\title{
\begin{tabular}{l|l} 
Mitraries & DSpace@MIT
\end{tabular}
}

\author{
MIT Open Access Articles
}

\section{NEW DETECTIONS OF ARSENIC, SELENIUM, AND OTHER HEAVY ELEMENTS IN TWO METAL-POOR STARS}

The MIT Faculty has made this article openly available. Please share how this access benefits you. Your story matters.

Citation: Roederer, Ian U., Hendrik Schatz, James E. Lawler, Timothy C. Beers, John J. Cowan, Anna Frebel, Inese I. Ivans, Christopher Sneden, and Jennifer S. Sobeck. "NEW DETECTIONS OF ARSENIC, SELENIUM, AND OTHER HEAVY ELEMENTS IN TWO METAL-POOR STARS.” The Astrophysical Journal 791, no. 1 (July 23, 2014): 32. (C) 2014 American Astronomical Society.

As Published: http://dx.doi.org/10.1088/0004-637x/791/1/32

Publisher: Institute of Physics/American Astronomical Society

Persistent URL: http://hdl.handle.net/1721.1/92932

Version: Final published version: final published article, as it appeared in a journal, conference proceedings, or other formally published context

Terms of Use: Article is made available in accordance with the publisher's policy and may be subject to US copyright law. Please refer to the publisher's site for terms of use. 


\title{
NEW DETECTIONS OF ARSENIC, SELENIUM, AND OTHER HEAVY ELEMENTS IN TWO METAL-POOR STARS*
}

\author{
IAn U. Roederer $^{1}$, Hendrik Schatz ${ }^{2,3,4}$, James E. Lawler ${ }^{5}$, Timothy C. Beers ${ }^{2,3,6}$, John J. Cowan ${ }^{7}$,

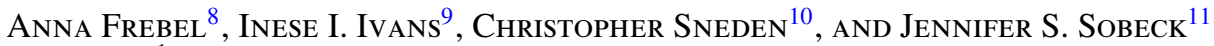 \\ ${ }^{1}$ Department of Astronomy, University of Michigan, Ann Arbor, MI 48109, USA \\ ${ }^{2}$ Department of Physics \& Astronomy, Michigan State University, East Lansing, MI 48824, USA \\ ${ }^{3}$ Joint Institute for Nuclear Astrophysics, Michigan State University, East Lansing, MI 48824, USA \\ ${ }^{4}$ National Superconducting Cyclotron Laboratory, Michigan State University, East Lansing, MI 48824, USA \\ ${ }^{5}$ Department of Physics, University of Wisconsin, Madison, WI 53706, USA \\ ${ }^{6}$ National Optical Astronomy Observatory, Tucson, AZ 85719, USA \\ ${ }^{7}$ Homer L. Dodge Department of Physics and Astronomy, University of Oklahoma, Norman, OK 73019, USA \\ ${ }^{8}$ Department of Physics \& Kavli Institute for Astrophysics and Space Research, \\ Massachusetts Institute of Technology, Cambridge, MA 02139, USA \\ ${ }^{9}$ Department of Physics and Astronomy, University of Utah, Salt Lake City, UT 84112, USA \\ ${ }^{10}$ Department of Astronomy, University of Texas at Austin, Austin, TX 78712, USA \\ ${ }^{11}$ Department of Astronomy, University of Virginia, Charlottesville, VA 22904, USA \\ Received 2014 April 7; accepted 2014 June 17; published 2014 July 23
}

\begin{abstract}
We use the Space Telescope Imaging Spectrograph on board the Hubble Space Telescope to obtain new high-quality spectra covering the $1900 \leqslant \lambda \leqslant 2360 \AA$ wavelength range for two metal-poor stars, HD 108317 and HD 128279 . We derive abundances of Cu II, Zn II, As I, Se I, Mo II, and Cd II, which have not been detected previously in either star. Abundances derived for Ge I, Te I, Os II, and Pt I confirm those derived from lines at longer wavelengths. We also derive upper limits from the non-detection of $\mathrm{W}$ II, $\mathrm{Hg}$ II, $\mathrm{Pb}$ II, and $\mathrm{Bi}$ I. The mean [As/Fe] ratio derived from these two stars and five others in the literature is unchanged over the metallicity range $-2.8<[\mathrm{Fe} / \mathrm{H}]<-0.6$, $\langle[\mathrm{As} / \mathrm{Fe}]\rangle=+0.28 \pm 0.14(\sigma=0.36 \mathrm{dex})$. The mean $[\mathrm{Se} / \mathrm{Fe}]$ ratio derived from these two stars and six others in the literature is also constant, $\langle[\mathrm{Se} / \mathrm{Fe}]\rangle=+0.16 \pm 0.09(\sigma=0.26 \mathrm{dex})$. The As and Se abundances are enhanced relative to a simple extrapolation of the iron-peak abundances to higher masses, suggesting that this mass region $(75 \leqslant A \leqslant 82$ ) may be the point at which a different nucleosynthetic mechanism begins to dominate the quasi-equilibrium $\alpha$-rich freezeout of the iron peak. $\langle[\mathrm{Cu}$ II $/ \mathrm{Cu}$ I $]\rangle=+0.56 \pm 0.23$ in HD 108317 and HD 128279 , and we infer that lines of $\mathrm{Cu}$ I may not be formed in local thermodynamic equilibrium in these stars. The [Zn/Fe], $[\mathrm{Mo} / \mathrm{Fe}],[\mathrm{Cd} / \mathrm{Fe}]$, and $[\mathrm{Os} / \mathrm{Fe}]$ ratios are also derived from neutral and ionized species, and each ratio pair agrees within the mutual uncertainties, which range from 0.15 to 0.52 dex.
\end{abstract}

Key words: nuclear reactions, nucleosynthesis, abundances - stars: abundances - stars: individual (HD 108317, HD 128279) - stars: Population II

Online-only material: color figures

\section{INTRODUCTION}

The near ultra-violet (NUV) portion of the spectra of latetype (FGK) stars contains absorption lines of atomic species that are not accessible in the optical or near-infrared domains. In the last two decades, NUV data obtained with the Goddard High Resolution Spectrograph and Space Telescope Imaging Spectrograph (STIS) on board the Hubble Space Telescope (HST) have enabled the first compelling detections of numerous elements heavier than the iron-group in late-type stars that are not chemically stratified. These elements include germanium (Ge, $Z=32$ ), arsenic (As, $Z=33$ ), selenium (Se, $Z=$ 34), cadmium ( $\mathrm{Cd}, Z=48)$, tellurium (Te, $Z=52)$, lutetium (Lu, $Z=71$ ), tantalum (Ta, $Z=73$ ), tungsten (W, $Z=74$ ), rhenium (Re, $Z=75)$, osmium (Os, $Z=76$ ), platinum $(\mathrm{Pt}$, $Z=78)$, gold (Au, $Z=79)$, and bismuth $(\mathrm{Bi}, Z=83$ ) (Cowan et al. 1996, 2002, 2005; Sneden et al. 1998, 2003; Roederer et al. 2009, 2010b, 2012a, 2012b; Barbuy et al.

\footnotetext{
* Based on observations made with the NASA/ESA Hubble Space Telescope, obtained at the Space Telescope Science Institute, which is operated by the Association of Universities for Research in Astronomy, Inc., under NASA contract NAS 5-26555. These observations are associated with programs GO-12268 and GO-12976.
}

2011; Peterson 2011; Roederer 2012; Roederer \& Lawler 2012; Siqueira Mello et al. 2013; Placco et al. 2014). These studies have also detected species not found in the optical domain, such as ionized molybdenum (Mo, $Z=42$ ); placed new upper limits on tin ( $\mathrm{Sn}, Z=50)$ and mercury $(\mathrm{Hg}, Z=80)$; and increased the number of useful abundance indicators of zirconium ( $\mathrm{Zr}$, $Z=40)$, iridium ( $\mathrm{Ir}, Z=77)$, and lead $(\mathrm{Pb}, Z=82)$. The high signal-to-noise ratio $(\mathrm{S} / \mathrm{N})$ and high spectral-resolution requirements for such studies limit $H S T$ to observing nearby, NUV-bright stars, so the number of stars where each of these heavy species have been studied rarely exceeds $\sim 5$.

When found in low-metallicity stars, these elements provide observational constraints on the production of rare, heavy nuclei in the earliest generations of stars. They provide critical evidence to locate the sites and constrain models of heavy-element nucleosynthesis via charged-particle, $r$-process, and $s$-process reactions. For example, in low-metallicity stars the abundance of $\mathrm{Ge}$ appears correlated with iron (Fe, $Z=26$; Cowan et al. 2005). Ge behaves like a primary element whose production may be linked with Fe. All previous observations of As and Se, however, only covered one star with $[\mathrm{Fe} / \mathrm{H}]<-2$ (Roederer 2012; Roederer \& Lawler 2012). There is no way to evaluate from these data whether As or Se correlate with Fe at low metallicity. 
Strontium ( $\mathrm{Sr}, Z=38$ ), the next heaviest element that is readily observable in low-metallicity stars, is not strongly correlated with Fe at low metallicity (e.g., Luck \& Bond 1985; McWilliam 1998; Travaglio et al. 2004). In the earliest generations of stars, these observations indicate that the lightest heavy nuclei whose production is decoupled from iron-group nucleosynthesis lie between $\mathrm{Ge}$ and $\mathrm{Sr}$, in the mass range $72 \leqslant A \leqslant 88$.

These studies also may confirm line identifications by corroborating abundances derived from multiple lines of the same species. The NUV domain of late-type stars exhibits many unidentified absorption lines. Abundances derived from one absorption feature must be confirmed by others to minimize the possibility of misidentification. Barbuy et al. (2011), Roederer \& Lawler (2012), and Roederer et al. (2012b) have made progress in this regard, but some detections remain unconfirmed (e.g., Cd I, Te I, Os II, and Ir II).

These studies also enable the detection of dominant ionization states (usually first ions) of elements whose minority species are detected in the optical domain. Nickel $(\mathrm{Ni}, Z=28)$, copper $(\mathrm{Cu}, Z=29)$, zinc $(\mathrm{Zn}, Z=30)$, and Mo are prominent examples (Peterson 2011; Roederer \& Lawler 2012; Wood et al. 2014). These observations provide valuable constraints on possible systematic effects, like over-ionization relative to the local thermodynamic equilibrium (LTE) populations, that could impact the minority species.

HST is currently the only source of high-resolution NUV spectra, so observing targets must be selected with care. Our previous observations (Roederer et al. 2012b) revealed striking similarities between two physically unrelated metal-poor giants, HD 108317 and HD 128279. These stars are first-ascent red giants with effective temperatures $\left(T_{\text {eff }} \approx 5100 \mathrm{~K}\right)$ and surface gravities $(\log g \approx 2.6$ in $\operatorname{cgs}$ units) that are identical within their uncertainties. They have nearly identical metallicities $([\mathrm{Fe} / \mathrm{H}] \approx-2.5)$ and light-element abundances. In contrast, the abundances of elements heavier than the iron-group in these two stars differ by a factor of $\approx 3$. Thus the spectra of these two stars are virtually identical except for the strength of lines of heavy elements. This fortuitous combination of characteristics simplifies the process of searching for lines of heavy elements and prompted us to acquire new STIS observations extending farther into the NUV. Here we present these new observations. We report new abundance data for several heavy elements and new constraints on the sources of systematic uncertainty discussed above.

Throughout this paper, we use the standard definitions of elemental abundances and ratios. For element $\mathrm{X}$, the logarithmic abundance is defined as the number of atoms of element $\mathrm{X}$ per $10^{12}$ hydrogen atoms, $\log \epsilon(\mathrm{X}) \equiv \log _{10}\left(N_{\mathrm{X}} / N_{\mathrm{H}}\right)+$ 12.0. For elements $\mathrm{X}$ and $\mathrm{Y}$, the logarithmic abundance ratio relative to the solar ratio of $\mathrm{X}$ and $\mathrm{Y}$ is defined as $[\mathrm{X} / \mathrm{Y}] \equiv \log _{10}\left(N_{\mathrm{X}} / N_{\mathrm{Y}}\right)-\log _{10}\left(N_{\mathrm{X}} / N_{\mathrm{Y}}\right)_{\odot}$. Abundances or ratios denoted with the ionization state indicate the total elemental abundance as derived from that particular ionization state. When reporting relative abundance ratios (e.g., $[\mathrm{X} / \mathrm{Fe}]$ ), these ratios compare neutrals with neutrals and ions with ions when possible.

\section{OBSERVATIONS}

In program GO-12976, we obtained new STIS observations (Kimble et al. 1998; Woodgate et al. 1998) of HD 108317 and HD 128279 using the E230M echelle grating, centered on $1978 \AA$, and the NUV Multianode Microchannel Array detector. The $0.06 \times 0$ '.2 slit yields a $\sim 2$ pixel resolving power
$(R \equiv \lambda / \Delta \lambda) \sim 30,000$. This setup produces wavelength coverage from $1610-2365 \AA$ in a single exposure, although in practice only wavelengths longer than $\approx 1900 \AA$ have useful $\mathrm{S} / \mathrm{N}$.

A total of 12 individual exposures of HD 108317 were scheduled over four visits from 2013 May 21 to 2013 July 15, resulting in a total exposure time of $28.6 \mathrm{ks}$. Fifteen exposures of HD 128279 were scheduled over three visits from 2013 January 22 to 2013 February 1, resulting in a total exposure time of $37.4 \mathrm{ks}$. The standard observational sequence includes acquisition and peak-up images to center the star on the narrow slit. These observations are reduced and calibrated using the calstis pipeline. After co-adding the individual exposures, the spectrum of each star has $\mathrm{S} / \mathrm{N}$ ranging from $\sim 10$ pixel $^{-1}$ near $1900 \AA$ to $\sim 50$ pixel $^{-1}$ near $2100 \AA$ and $\gtrsim 100$ pixel $^{-1}$ at $2350 \AA$.

STIS observations of HD 108317 and HD 128279 obtained previously in program GO-12268 covered the NUV spectral range from 2280 to $3115 \AA$. We co-add those observations with our new observations in the region of overlap. Final $\mathrm{S} / \mathrm{N}$ in the region from 2280 to $2365 \AA$ are $\gtrsim 130$ pixel $^{-1}$.

\section{MODEL ATMOSPHERES AND STELLAR PARAMETERS}

We adopt the same ATLAS9 model atmospheres (Castelli $\&$ Kurucz 2004) used in our previous study. For HD 108317, $T_{\text {eff }}, \log g$, microturbulent velocity $\left(v_{t}\right)$, and metallicity $([\mathrm{M} / \mathrm{H}])$ of the model atmosphere are $5100 \pm 200 \mathrm{~K}, 2.67 \pm 0.2$, $1.50 \pm 0.2 \mathrm{~km} \mathrm{~s}^{-1}$, and $-2.37 \pm 0.20$, respectively. For HD $128279, T_{\text {eff }}, \log g, v_{t}$, and $[\mathrm{M} / \mathrm{H}]$ are $5080 \pm 200 \mathrm{~K}$, $2.57 \pm 0.2,1.60 \pm 0.2 \mathrm{~km} \mathrm{~s}^{-1}$, and $-2.46 \pm 0.22$, respectively.

Here we briefly summarize the methods Roederer et al. (2012b) used to derive these parameters. The Alonso et al. (1999) color-temperature relations were used to calculate an initial estimate for $T_{\text {eff }}$ from the dereddened $V-K$ color. The $\log g$ values were calculated from these temperatures and Hipparcos parallax measurements, assuming a mass of $0.8 \pm$ $0.1 M_{\odot}$. Microturbulence velocities were derived by minimizing correlations between line strength and the abundances derived from optical Fe I lines. The initial $T_{\text {eff }}$ values were revised to minimize correlations between the excitation potentials (E.P.) and the abundances derived from optical Fe I lines. An analysis of interstellar absorption lines toward each star supported the adjusted $T_{\text {eff }}$ values. Revised $\log g$ values were calculated accordingly. Finally, the metallicity of the model atmosphere was adopted to be equivalent to the Fe abundance derived from Fe II lines. These steps were iterated until convergence $\left(T_{\text {eff }}\right.$ stable within $10 \mathrm{~K}, \log g$ within $0.01 \mathrm{dex}, v_{t}$ within $0.05 \mathrm{~km} \mathrm{~s}^{-1}$, and $[\mathrm{M} / \mathrm{H}]$ within $0.01 \mathrm{dex}$ ).

\section{IRON ABUNDANCE TRENDS WITH WAVELENGTH}

In our previous work on HD 108317 and HD 128279 (Roederer et al. 2012b), we uncovered evidence that Fe I lines did not yield consistent abundances over the NUV and optical spectral ranges. Specifically, abundances derived from lines with $\lambda<4000 \AA$ were lower than abundances derived from lines with longer wavelengths. The most severe deficiency in HD 108317 and HD 128279 occurred for lines with $3100<\lambda<3647 \AA$, as illustrated in Figure 9 of Roederer et al. Subsequent studies by Lawler et al. (2013) and Wood et al. (2013) of NUV and optical Fe I, Ti I, and Ti II lines revealed similar inconsistencies in the metal-poor subgiant HD 84937. However, Wood et al. (2014) did not find any such inconsistencies in their analysis of Ni I lines in this star. In an independent analysis of the metal-poor 
Table 1

Iron Equivalent Widths and Abundances

\begin{tabular}{|c|c|c|c|c|c|c|c|c|}
\hline \multirow[b]{2}{*}{ Species } & \multirow[b]{2}{*}{$\begin{array}{c}\lambda \\
(\AA)\end{array}$} & \multirow[b]{2}{*}{$\begin{array}{l}\text { E.P. } \\
(\mathrm{eV})\end{array}$} & \multirow[b]{2}{*}{$\log g f$} & \multirow[b]{2}{*}{$\begin{array}{c}\text { NIST } \\
\text { Grade }^{\mathrm{a}}\end{array}$} & \multicolumn{2}{|c|}{ HD 108317} & \multicolumn{2}{|c|}{ HD 128279} \\
\hline & & & & & $\begin{array}{l}\text { Eq. Wid. } \\
\text { (mÅ) }\end{array}$ & $\log \epsilon$ & 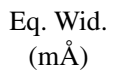 & $\log \epsilon$ \\
\hline $\mathrm{Fe}_{\mathrm{I}}$ & 2132.02 & 0.00 & -1.33 & $\mathrm{C}+$ & $\ldots$ & $\ldots$ & 90.1 & 4.44 \\
\hline $\mathrm{Fe}_{\mathrm{I}}$ & 2145.19 & 0.05 & -1.56 & $\mathrm{C}$ & 79.8 & 4.56 & 82.2 & 4.56 \\
\hline $\mathrm{Fe} I$ & 2161.58 & 0.11 & -1.76 & $\mathrm{D}+$ & 83.0 & 4.89 & 86.1 & 4.89 \\
\hline $\mathrm{Fe}_{\mathrm{I}}$ & 2176.84 & 0.12 & -1.66 & $\mathrm{C}+$ & $\ldots$ & $\ldots$ & 91.4 & 4.90 \\
\hline $\mathrm{Fe}_{\mathrm{I}}$ & 2191.20 & 0.12 & -1.80 & $\mathrm{C}$ & 72.0 & 4.65 & 70.0 & 4.51 \\
\hline $\mathrm{Fe} I$ & 2196.04 & 0.11 & -0.59 & $\mathrm{C}+$ & 144.2 & 4.45 & 156.3 & 4.51 \\
\hline $\mathrm{Fe}_{\mathrm{I}}$ & 2200.72 & 0.11 & -0.99 & $\mathrm{C}$ & $\ldots$ & $\ldots$ & 140.2 & 4.79 \\
\hline $\mathrm{Fe}_{\mathrm{I}}$ & 2228.17 & 0.05 & -2.11 & $\mathrm{D}+$ & 81.4 & 5.11 & 79.5 & 5.00 \\
\hline $\mathrm{Fe}_{\mathrm{I}}$ & 2259.28 & 0.05 & -2.31 & $\mathrm{C}$ & 60.2 & 4.62 & $\ldots$ & $\ldots$ \\
\hline $\mathrm{Fe}_{\mathrm{I}}$ & 2259.51 & 0.00 & -1.32 & $\mathrm{~B}+$ & $\ldots$ & $\ldots$ & 187.4 & 5.32 \\
\hline $\mathrm{Fe}$ I & 2265.05 & 0.05 & -2.11 & $\mathrm{C}$ & 96.3 & 5.38 & 91.0 & 5.24 \\
\hline $\mathrm{Fe}_{\mathrm{I}}$ & 2267.08 & 0.05 & -1.75 & B & $\ldots$ & $\ldots$ & 91.8 & 4.88 \\
\hline $\mathrm{Fe}_{\mathrm{I}}$ & 2275.19 & 0.11 & -2.32 & $\mathrm{D}+$ & 71.3 & 5.07 & 69.3 & 4.93 \\
\hline $\mathrm{Fe} I$ & 2283.30 & 0.12 & -2.22 & $\mathrm{~B}+$ & 92.7 & 5.50 & $\ldots$ & $\ldots$ \\
\hline $\mathrm{Fe} I$ & 2294.41 & 0.11 & -1.54 & B & 83.6 & 4.63 & 86.0 & 4.62 \\
\hline $\mathrm{Fe}_{\mathrm{I}}$ & 2297.79 & 0.05 & -1.10 & $\mathrm{D}+$ & 96.7 & 4.37 & 113.3 & 4.56 \\
\hline $\mathrm{Fe} I$ & 2298.66 & 0.11 & -2.42 & $\mathrm{C}$ & 82.5 & 5.48 & $\ldots$ & $\ldots$ \\
\hline $\mathrm{Fe} I$ & 2299.22 & 0.09 & -1.55 & $\mathrm{C}$ & 76.4 & 4.43 & 85.2 & 4.57 \\
\hline $\mathrm{Fe}_{\mathrm{I}}$ & 2309.00 & 0.11 & -1.39 & $\mathrm{C}$ & 106.0 & 4.97 & 118.7 & 5.09 \\
\hline Fe II & 2122.45 & 1.96 & -2.59 & $\mathrm{D}+$ & 41.2 & 5.06 & 42.4 & 5.00 \\
\hline Fe II & 2162.02 & 1.96 & -0.75 & $\mathrm{C}$ & 87.2 & 4.90 & 88.7 & 4.85 \\
\hline Fe II & 2209.03 & 4.77 & -0.13 & $\mathrm{C}+$ & $\ldots$ & $\ldots$ & 28.6 & 4.98 \\
\hline Fe II & 2251.55 & 0.05 & -2.35 & $\mathrm{D}$ & 156.8 & 5.03 & 159.9 & 5.00 \\
\hline Fe II & 2254.41 & 0.11 & -3.07 & $\mathrm{D}$ & 78.0 & 4.83 & $\ldots$ & $\ldots$ \\
\hline Fe II & 2260.86 & 0.11 & -2.00 & $\mathrm{C}+$ & 145.4 & 4.84 & 142.6 & 4.75 \\
\hline Fe II & 2262.69 & 0.11 & -2.22 & $\mathrm{C}+$ & 126.0 & 4.71 & 134.5 & 4.72 \\
\hline Fe II & 2268.56 & 0.12 & -2.73 & $\mathrm{C}$ & 104.5 & 5.16 & 103.1 & 5.05 \\
\hline Fe II & 2268.82 & 0.05 & -2.61 & $\mathrm{C}+$ & 114.7 & 5.02 & 118.2 & 4.99 \\
\hline Fe II & 2351.20 & 2.66 & -0.23 & $\mathrm{C}+$ & 97.2 & 5.23 & 96.0 & 5.14 \\
\hline Fe II & 2351.67 & 5.25 & -0.05 & $\mathrm{C}$ & $\ldots$ & $\ldots$ & 21.3 & 5.14 \\
\hline
\end{tabular}

Note. ${ }^{a}$ NIST grades correspond to accuracies of $\leqslant 7 \%(B+), 10 \%(B), 18 \%(C+), 25 \%(C), 40 \%(D+)$, and $50 \%(D)$.

giant BD+44 493 by Ito et al. (2013; see also Placco et al. 2014), the Fe I lines with $\lambda<3700 \AA$ yield a mean abundance about 0.15 dex lower than the Fe I lines with $\lambda>3700 \AA$, as shown by their Figure 6 .

These studies investigated several possible causes of the abundance discrepancies. The wavelength region most severely affected is that around the transition from the Paschen continuum to the Balmer continuum. Roederer et al. (2012b) concluded that a missing source of continuous opacity could account for part of the effect. Lawler et al. (2013) and Wood et al. $(2013,2014)$ suggested that departures from LTE could also be partly responsible. Missing opacity would impact all species with transitions in the affected wavelength range equally, while non-LTE effects would affect each transition individually, if at all. Ultimately, Roederer et al. adopted an empirical set of wavelength-dependent abundance corrections for all transitions based on the results from $\mathrm{Fe}$ I lines.

Our new STIS data extend to even shorter wavelengths. We measure equivalent widths of relatively unblended $\mathrm{Fe}_{\mathrm{I}}$ and Fe II lines in our data. All $\log g f$ values are adopted from the National Institute of Standards and Technology (NIST) Atomic Spectra Database (ASD; Kramida et al. 2013). This critical compilation assesses the accuracy of these $\log g f$ values to be $0.03-0.30$ dex (grades B+ to D). The wavelengths, E.P., $\log g f$ values, estimated accuracies, and equivalent widths are presented in Table 1.
We derive the abundances using a recent version of the line analysis code MOOG (Sneden 1973) that includes the contribution of Rayleigh scattering from $\mathrm{HI}$ in the source function, as discussed by Sobeck et al. (2011). Figure 1 illustrates the abundances derived from lines of $\mathrm{Fe}$ I and $\mathrm{Fe}$ II as a function of wavelength. New abundances for lines with $\lambda<2360 \AA$ are shown, together with the data for lines at longer wavelengths from Roederer et al. (2012b). All abundances in Figure 1 are referenced to the mean $\mathrm{Fe}$ abundance derived from lines with $\lambda>4400 \AA$. The line-to-line dispersion for Fe I and Fe II lines at short wavelengths in both stars is significantly larger than for lines at longer wavelengths. The abundance "dip" found by Roederer et al. for lines with $3100<\lambda<4000 \AA$ exceeds the dispersion of $\mathrm{Fe}$ I at these wavelengths. No dip is apparent for Fe II lines, but the number of lines in the affected region is small.

To eliminate the possibility that uncertainties in the $\log g f$ values are a source of this error, we show the line-by-line differential abundances between HD 108317 and HD 128279 in Figure 2. For Fe I, the dispersion for lines with $\lambda<3100 \AA$, 0.093 dex, is two times larger than the dispersion for lines with $\lambda>3500 \AA$, 0.045 dex. For Fe II, the dispersion for lines with $\lambda<3100 \AA$, $0.090 \mathrm{dex}$, is also several times larger than the dispersion for lines with $\lambda>3500 \AA$, 0.034 dex. The $\mathrm{S} / \mathrm{N}$ are $\gg 100$ for the STIS, HIRES, and MIKE spectra used from $2350 \AA$ to $6800 \AA$, and the $\mathrm{S} / \mathrm{N}$ are $>50$ from $2100 \AA$ to $2350 \AA$. 

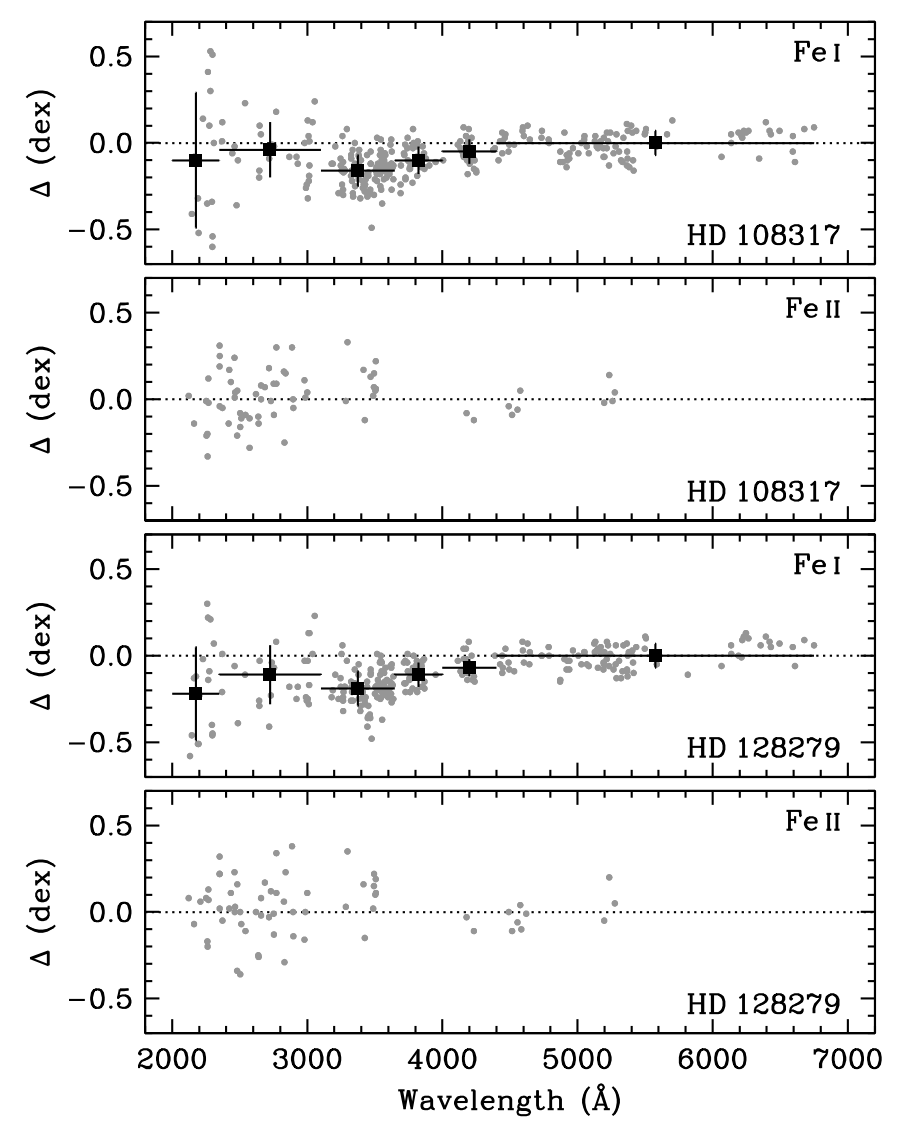

Figure 1. Relative Fe abundances from Fe I and II lines. Gray dots represent abundances derived from individual lines. Large black squares represent the mean abundance in each wavelength range. Horizontal error bars mark the wavelength range, and vertical error bars indicate the standard deviation. The dotted line in each panel marks the mean abundance derived from lines with $\lambda>4400 \AA$.

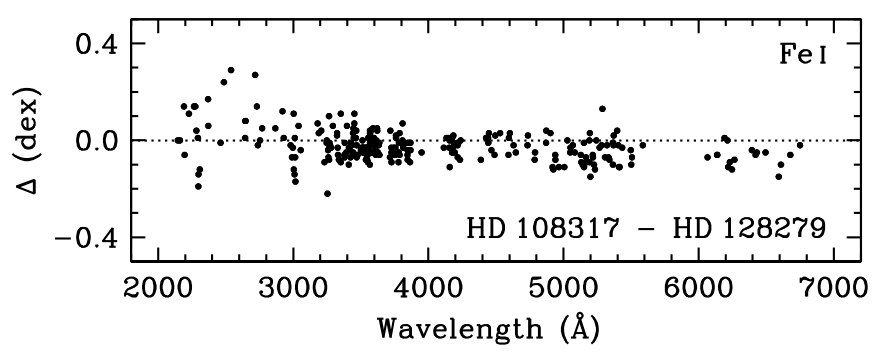

Figure 2. Line-by-line differences in the Fe I abundances. The differences are in the sense of HD 108317 minus HD 128279. The dotted line indicates a difference of zero. These differences are independent of the choice of $\log g f$ values.

This demonstrates the challenge of deriving reliable abundances from crowded regions of spectra. This also suggests the presence of a threshold in abundance precision from being limited by $\mathrm{S} / \mathrm{N}$ to being limited by line blends, continuum placement, etc. In principle, significantly higher spectral resolution $(R \gtrsim$ $60,000)$ would help to identify the continuum level and signal the presence of blending lines.

Lacking further information about how to properly treat each species of each element, we follow Roederer et al. (2012b) in adopting a set of wavelength-dependent abundance corrections for all transitions based on the results from $\mathrm{Fe}_{\mathrm{I}}$ lines. The effect of these corrections is to adopt a local metallicity in hopes of preserving accurate ratios of one element to another. Our adopted corrections are listed in Table 2. For completeness,
Table 2

Mean Fe I Abundances Binned by Wavelength

\begin{tabular}{lcccc}
\hline \hline $\begin{array}{l}\text { Wavelength } \\
\text { Range }(\AA)\end{array}$ & $\langle\log \epsilon\rangle$ & Std. Dev. & $N$ & Correction \\
\hline \multicolumn{5}{c}{ HD 108317} \\
\hline$<360$ & 4.87 & 0.39 & 9 & +0.10 \\
$2360-3100$ & 4.93 & 0.16 & 32 & +0.04 \\
$3100-3647$ & 4.81 & 0.09 & 107 & +0.16 \\
$3647-4000$ & 4.87 & 0.08 & 40 & +0.10 \\
$4000-4400$ & 4.92 & 0.07 & 27 & +0.05 \\
$4400-6750$ & 4.97 & 0.07 & 94 & $\equiv 0.0$ \\
\hline & & HD 128279 & & \\
\hline$<2360$ & 4.80 & 0.27 & 10 & +0.22 \\
$2360-3100$ & 4.91 & 0.17 & 28 & +0.11 \\
$3100-3647$ & 4.83 & 0.10 & 96 & +0.19 \\
$3647-4000$ & 4.91 & 0.07 & 32 & +0.11 \\
$4000-4400$ & 4.97 & 0.07 & 19 & +0.05 \\
$4400-6750$ & 5.02 & 0.07 & 91 & $\equiv 0.0$ \\
\hline
\end{tabular}

Table 2 also lists the corrections for longer wavelengths from Table 10 of Roederer et al.

\section{HEAVY ELEMENT LINE DETECTION}

We follow the same approach as Roederer et al. (2012b) to identify reliable abundance indicators of heavy elements in the NUV spectra of HD 108317 and HD 128279. We search for transitions reported in recent studies by Peterson (2011), Roederer \& Lawler (2012), Roederer et al. (2012b), and Siqueira Mello et al. (2013). We search for leading lines in the NUV that are listed in recent laboratory or theoretical studies of heavy element spectra (Den Hartog et al. 2005; Nilsson et al. 2008). We also overplot the high S/N spectra of HD 108317 and HD 128279 and search for significant differences by eye. As demonstrated by Roederer et al. (2012a, 2012b), these differences frequently indicate the presence of heavy element absorption lines in HD 108317. We then generate a synthetic spectrum for each line. Many candidates are too weak or too blended to yield a secure detection. Figures 3 and 4 illustrate sections of the STIS spectra around 17 lines that are detected in one or both stars.

\section{ABUNDANCE ANALYSIS OF HEAVY ELEMENTS}

The atomic data for lines of interest are listed in Table 3. References and uncertainties for the $\log g f$ values are noted. We use MOOG to generate synthetic spectra, and we derive abundances or upper limits by spectrum-synthesis matching. We include hyperfine splitting (hfs) of As I, Cd II, Yb II, and $\mathrm{Hg}$ II. Isotope shifts (IS) are included in our syntheses of Cd II, Yb II, and $\mathrm{Hg}$ II, and we use the $r$-process isotope mixtures presented by Sneden et al. (2008). The complete hfs and IS line component patterns for these transitions are presented in Tables 4, 8, 10, and 12 of Roederer \& Lawler (2012).

Several examples of key lines of interest in HD 108317 are illustrated in Figure 5. Our procedure for generating the line lists and producing acceptable matches to the observed spectra is described in detail in Roederer et al. (2012b). We briefly summarize these methods here. There are numerous unidentified lines in NUV spectra of low-metallicity late-type stars, and the transition probabilities for many identified lines are not known with sufficient accuracy to produce acceptable matches between the observed and synthetic spectra without additional effort. 

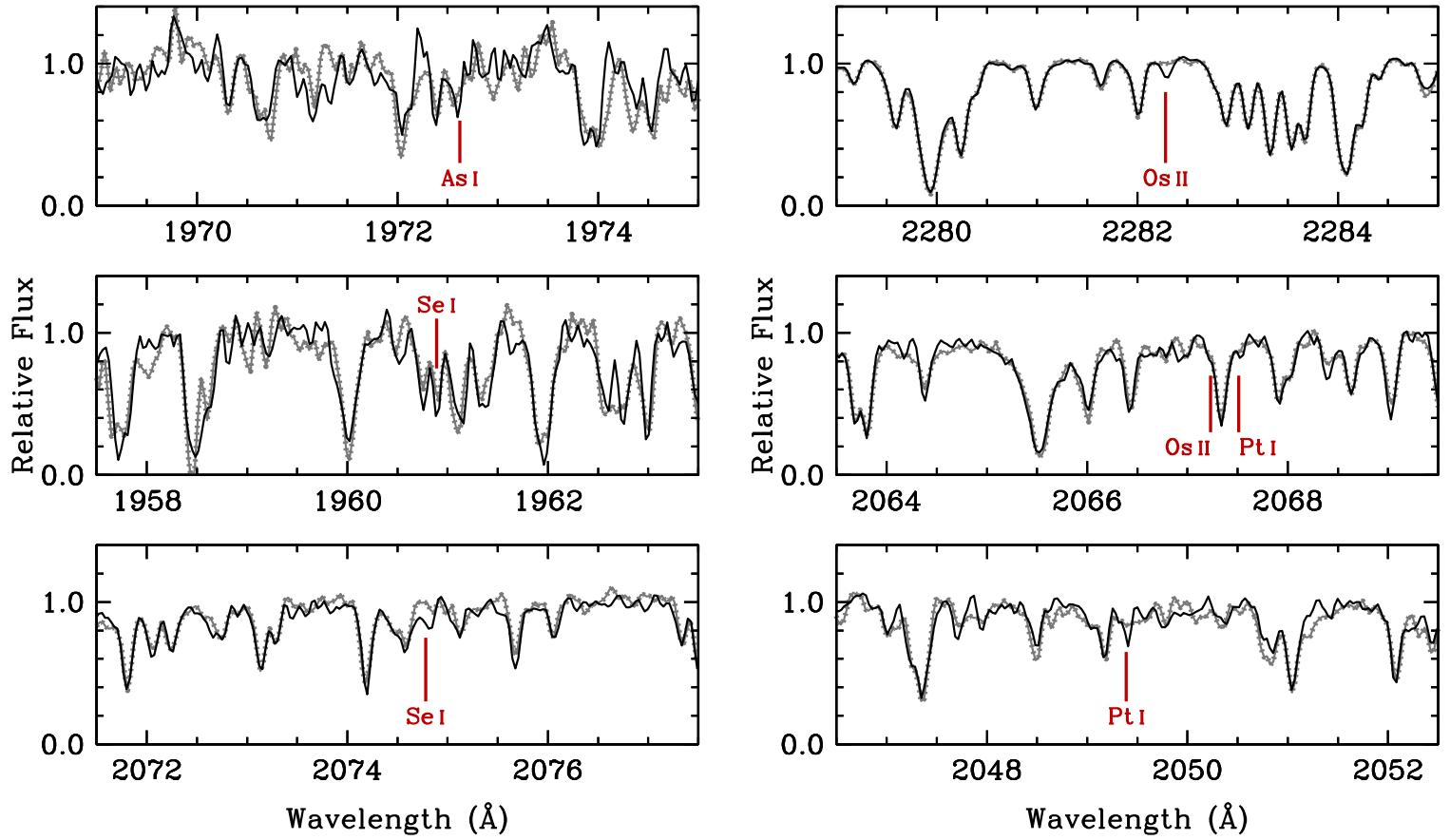

Figure 3. Spectra of HD 108317 (thin black curve) and HD 128279 (studded gray curve) around several lines of interest. Air wavelengths are shown for data with $\lambda>2000 \AA$, and vacuum wavelengths are shown for data with $\lambda<2000 \AA$. Note the similarity between the spectra of these two stars except at the wavelengths of absorption features arising from heavy elements.

(A color version of this figure is available in the online journal.)
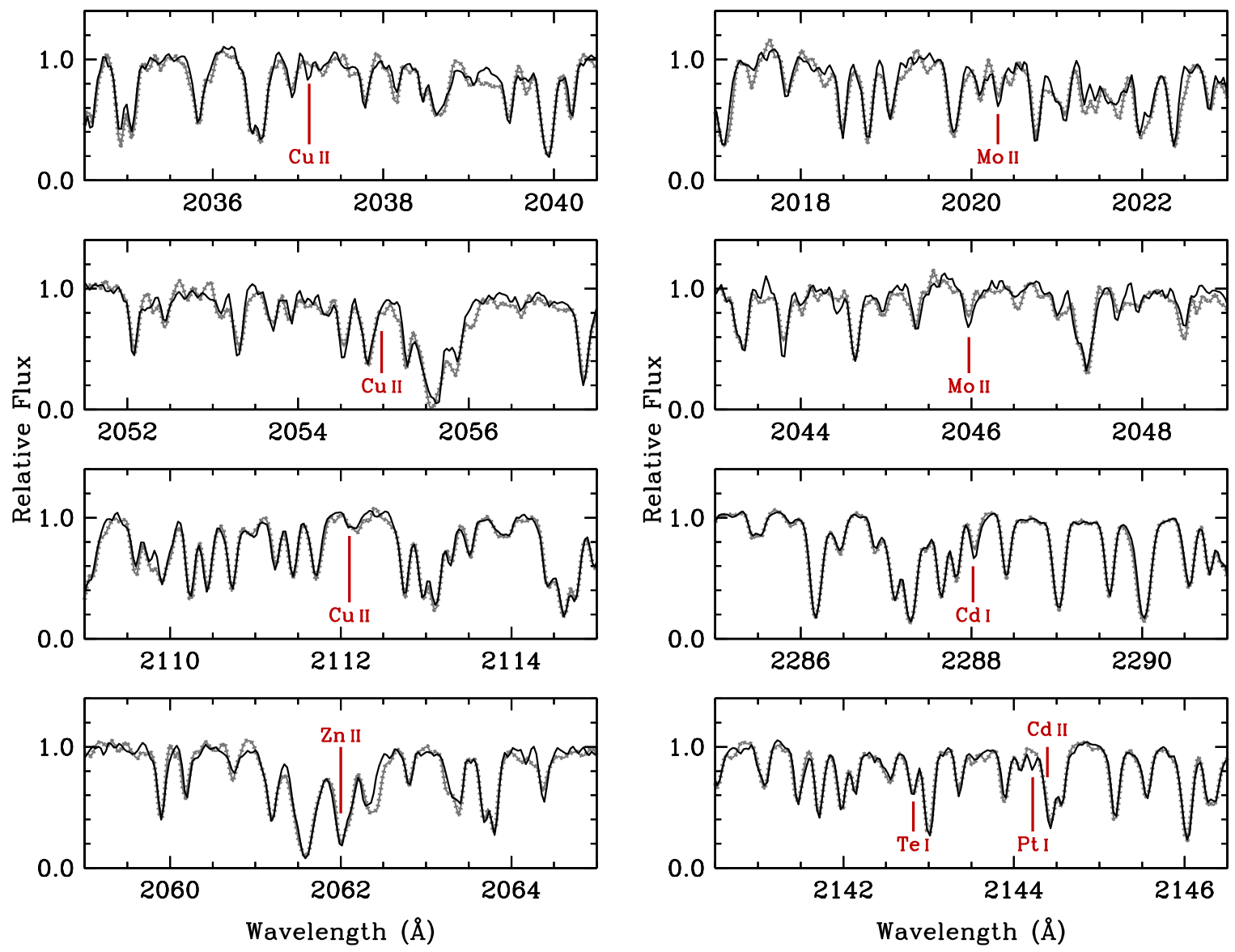

Figure 4. Spectra of HD 108317 (thin black curve) and HD 128279 (studded gray curve) around several lines of interest. As in Figure 3, note the similarity between the spectra of these two stars except at the wavelengths of absorption features arising from heavy elements.

(A color version of this figure is available in the online journal.) 
Table 3

Atomic Data and Line Abundances

\begin{tabular}{|c|c|c|c|c|c|c|c|}
\hline Species & $Z$ & $\begin{array}{l}\text { Wavelength }^{\mathrm{a}} \\
(\AA)\end{array}$ & $\begin{array}{l}\text { E.P. } \\
(\mathrm{eV})\end{array}$ & $\log g f(\sigma)$ & Ref. & $\begin{array}{c}\log \epsilon(\sigma) \\
\text { HD } 108317\end{array}$ & $\begin{array}{c}\log \epsilon(\sigma) \\
\text { HD } 128279\end{array}$ \\
\hline $\mathrm{Cu}$ II & 29 & 2037.13 & 2.83 & $-0.23(0.03)$ & 1 & $+1.48(0.15)$ & $+1.21(0.30)$ \\
\hline $\mathrm{Cu}$ II & 29 & 2054.98 & 2.93 & $-0.29(0.03)$ & 1 & $+1.57(0.20)$ & $+1.45(0.30)$ \\
\hline $\mathrm{Cu}$ II & 29 & 2112.10 & 3.25 & $-0.11(0.10)$ & 1 & $+1.24(0.20)$ & $+1.42(0.30)$ \\
\hline $\mathrm{Cu}$ II & 29 & 2126.04 & 2.83 & $-0.23(0.07)$ & 1 & $+1.39(0.20)$ & $\ldots$ \\
\hline Zn II & 30 & 2062.00 & 0.00 & $-0.29(0.04)$ & 2 & $+2.50(0.30)$ & $+2.38(0.30)$ \\
\hline $\mathrm{Ge}_{\mathrm{I}}$ & 32 & 2065.21 & 0.07 & $-0.79(0.10)$ & 3 & $<+0.85$ & $\ldots$ \\
\hline As I & 33 & 1972.62 & 0.00 & $-0.63(0.05)$ & 1 & $-0.30(0.40)$ & $-0.49(0.40)$ \\
\hline Se I & 34 & 1960.89 & 0.00 & $-0.43(0.08)$ & 4 & $+1.30(0.40)$ & $+0.57(0.40)$ \\
\hline Se I & 34 & 2074.78 & 0.00 & $-2.26(0.03)$ & 4 & $+1.25(0.40)$ & $+0.53(0.40)$ \\
\hline Mo II & 42 & 2015.11 & 0.00 & $-0.49(0.11)$ & 5 & $\ldots$ & $-0.74(0.30)$ \\
\hline Mo II & 42 & 2020.31 & 0.00 & $+0.02(0.10)$ & 5 & $-0.16(0.30)$ & $-0.51(0.20)$ \\
\hline Mo II & 42 & 2045.97 & 0.00 & $-0.35(0.12)$ & 5 & $+0.18(0.20)$ & $-0.48(0.20)$ \\
\hline $\mathrm{Cd}_{\mathrm{I}}$ & 48 & 2288.02 & 0.00 & $+0.15(0.02)$ & 4 & $-1.21(0.20)$ & $-1.34(0.30)$ \\
\hline Cd II & 48 & 2144.39 & 0.00 & $+0.02(0.02)$ & 1 & $-1.08(0.30)$ & $-1.30(0.30)$ \\
\hline Te I & 52 & 2142.82 & 0.00 & $-0.32(0.08)$ & 6 & $+0.14(0.30)$ & $-0.04(0.30)$ \\
\hline Yb II & 70 & 2116.68 & 0.00 & $-1.34(0.10)$ & 1 & $<-0.50$ & $<-0.98$ \\
\hline W II & 74 & 2088.20 & 0.39 & $-0.02(0.03)$ & 7 & $<-0.80$ & $<-0.58$ \\
\hline W II & 74 & 2118.88 & 0.00 & $-0.77(0.05)$ & 7 & $<-0.40$ & $<-0.68$ \\
\hline Os II & 76 & 2067.23 & 0.45 & $-0.05(0.03)$ & 8 & $-0.47(0.30)$ & $<-0.38$ \\
\hline Os II & 76 & 2282.28 & 0.00 & $-0.05(0.04)$ & 9 & $-0.83(0.15)$ & $<-1.28$ \\
\hline$P t I$ & 78 & 2049.39 & 0.00 & $+0.02(0.06)$ & 1 & $-0.42(0.20)$ & $<-0.53$ \\
\hline Pt I & 78 & 2067.51 & 0.00 & $-0.62(0.03)$ & 10 & $+0.01(0.30)$ & $<+0.02$ \\
\hline Pt I & 78 & 2144.21 & 0.00 & $-0.37(0.07)$ & 11 & $-0.37(0.20)$ & $<-0.78$ \\
\hline $\mathrm{Hg}_{\text {II }}$ & 80 & $1942.27^{b}$ & 0.00 & $-0.40(0.04)$ & 1 & $<+0.30$ & $<+0.53$ \\
\hline $\mathrm{Pb}$ II & 82 & 2203.53 & 1.75 & $-0.14(0.10)$ & 3 & $<+0.09$ & $<+0.12$ \\
\hline $\mathrm{Bi}$ I & 83 & 2230.61 & 0.00 & $+0.02(0.06)$ & 12 & $<-1.02$ & $\ldots$ \\
\hline
\end{tabular}

Notes.

a Air wavelengths are given for $\lambda>2000 \AA$ and vacuum values below.

b The wavelength of this line was incorrectly listed in Table 14 of Roederer \& Lawler (2012). The value given here is correct.

References. (1) Roederer \& Lawler 2012; (2) Bergeson \& Lawler 1993; (3) NIST; (4) Morton 2000; (5) Sikström et al. 2001; (6) Roederer et al. 2012a; (7) Nilsson et al. 2008; (8) Ivarsson et al. 2004; (9) Quinet et al. 2006; (10) Den Hartog et al. 2005; (11) This study; (12) Morton 2000.

Transition probabilities measured by laboratory experiments are used in our syntheses whenever possible. Unidentified lines are fit assuming the absorption arises from Fe I lines with a lower E.P. of $1.5 \mathrm{eV}$, and we use the observed line profile to constrain the strength of each line. Lines of interest must be distinct from such lines to merit use as abundance indicators.

\section{COMMENTS ON INDIVIDUAL ELEMENTS}

In this section, we discuss the detection of multiple lines as a way to confirm the line identifications for Te, Ir, and Pt. We also discuss the limitations of line identification for $\mathrm{Pb}$ and $\mathrm{Bi}$.

\subsection{Tellurium}

Although two Te I lines have been used as abundance indicators previously, the spectra of the four stars studied by Roederer \& Lawler (2012) and Roederer et al. (2012a) did not cover both lines simultaneously in any of these stars. Now, our combined NUV observations of HD 108317 and HD 128279 cover both Te I lines ( 2142 and $2385 \AA$ ) in these two stars. The abundances derived from these lines agree for each star. In HD 108317 the Te I 2142 and $2385 \AA$ lines yield $\log \epsilon(\mathrm{Te})=+0.14 \pm 0.30$ and $+0.02 \pm 0.25$, and in HD 128279 these lines yield $\log \epsilon$ $(\mathrm{Te})=-0.04 \pm 0.30$ and $-0.18 \pm 0.25$. This increases our confidence that both detections are legitimate and that both lines are reliable abundance indicators.

\subsection{Iridium}

A line of Ir II at $2126 \AA$ has been used previously by Roederer \& Lawler (2012) as an abundance indicator in HD 160617. This line also appears in our spectra of HD 108317 and HD 128279. Its presence in HD 128279 is surprising, since no absorption lines of the neighboring elements Os or Pt are detected in HD 128279. If we assume that the absorption at $2126.81 \AA$ in HD 128279 is due to Ir II, we derive an Ir abundance $(\log \epsilon \approx$ $-0.3 \pm 0.3)$ that is 1.0 dex higher than the upper limit on Os $(\log \epsilon<-1.28)$ and 0.5 dex higher than the upper limit on $\mathrm{Pt}(\log \epsilon<-0.78)$. This suggests that the absorption at $2126.81 \AA$ is probably not due to Ir II in HD 108317 or HD 128279. Instead, this absorption can be fit by increasing the $\log g f$ value of the $\mathrm{Fe}_{\mathrm{I}}$ line at $2126.82 \AA$. Conservatively, we assume that the absorption detected in HD 160617 is also not due to Ir II. The Ir abundances reported in Tables 14 and 15 of Roederer \& Lawler should be regarded as upper limits.

\subsection{Platinum}

Roederer \& Lawler (2012) derived Pt abundances from the Pt I 2049.39 and 2067.51 $\AA$ lines in HD 160617, and we also detect these lines in HD 108317. One new line of Pt I, 2144.21 $\AA$, is also detected in HD 108317 and listed in Table 3. Den Hartog et al. (2005) measured the radiative lifetime of the upper level of this transition $\left[5 d^{8} 6 s 6 p\left({ }^{2} F\right)^{3} D ; 46622.489 \mathrm{~cm}^{-1}\right]$. The $2144.21 \AA$ line is a dominant $(68 \%)$ decay branch to the ground level 

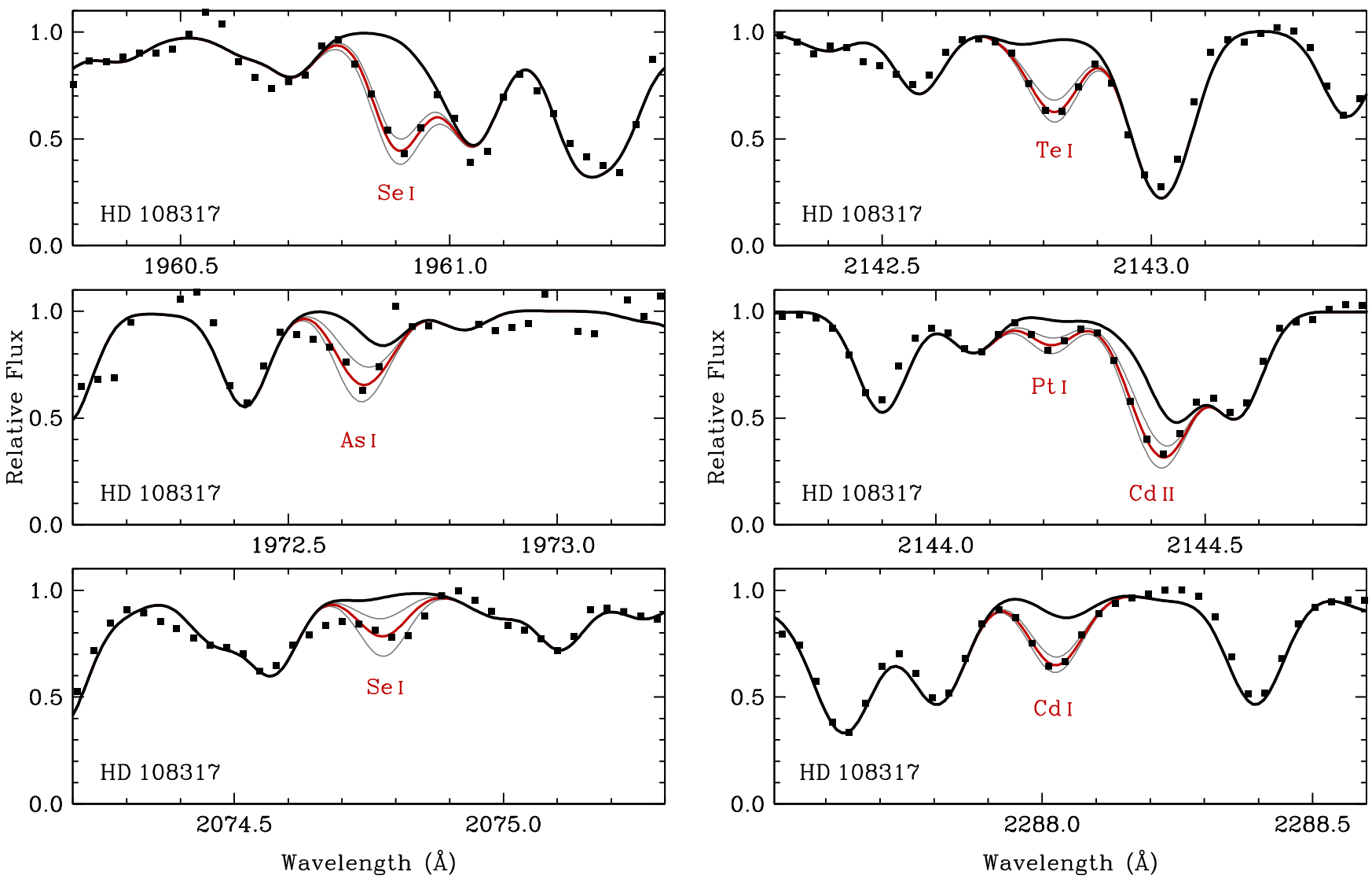

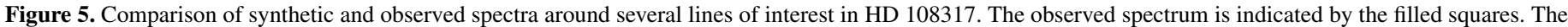

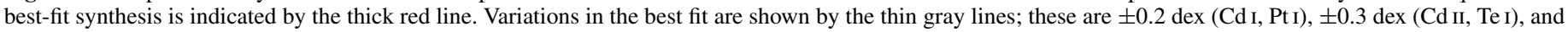
\pm 0.4 dex (As I, Se I). The thick black lines represent a syntheses with no absorption from the line(s) of interest.

(A color version of this figure is available in the online journal.)

$\left(4 d^{9} 6 s^{3} D_{3}\right)$. We use these measurements to calculate the $\log g f$ value of this line, $-0.37 \pm 0.07$. The abundances derived from all three Pt I lines are in agreement, as shown in Table 3. They are also in agreement with the abundances derived from two Pt I lines at longer wavelengths in HD 108317, listed in Table 3 of Roederer et al. (2012b).

\subsection{Lead}

Only a few lines of neutral $\mathrm{Pb}$ have been detected in the optical and NUV spectral range, and these are usually blended with other features that complicate attempts to secure a reliable detection. Neutral $\mathrm{Pb}$ never comprises more than a few percent of all $\mathrm{Pb}$ atoms in the conditions found in latetype stellar atmospheres (Mashonkina et al. 2012; Roederer \& Lawler 2012). Mashonkina et al. investigated the effect of overionization on $\mathrm{Pb}$ I lines and presented a set of non-LTE corrections for abundances derived from $\mathrm{Pb}$ I lines. For the Pb I $4057 \AA$ line in HD 108317, their calculations suggest the correction is +0.52 dex.

Ionized $\mathrm{Pb}$ is even more elusive. The NIST database lists only eight classified Pb II transitions from 2000 to $10000 \AA$, and only one of these transitions originates from an electronic level below $7 \mathrm{eV}$. The $2203.53 \AA$ transition connects the upper $6 s^{2} 7 s^{2} S_{1 / 2}$ level to the lower $6 s^{2} 6 p^{2} P_{3 / 2}^{o}$ level. It is difficult to estimate the accuracy of the $\log g f$ value of this line, which Morton (2000) reports based on calculations by Migdałek (1983).

A small amount of absorption is detected at the predicted wavelength of the $\mathrm{Pb}$ II line, 2203.53 $\AA$. As shown in Figure 6, this absorption falls on the red wing of a stronger, unidentified feature with wavelength $2203.44 \AA$. A weak Fe I line also shows absorption in the blue wing of this unidentified feature. We generate a fictitious line at $2203.44 \AA$ in our synthesis to reproduce the observed line profile in both HD 108317 and HD 128279, yet residual absorption at the predicted wavelength of the $\mathrm{Pb}$ II line persists. As shown in Figure 6, this absorption is present in both HD 108317 and HD 128279. Roederer et al. (2012b) found that none of the elements at or beyond the 3rd $r$-process peak could be detected in HD 128279. It would be uncharacteristic of the heavy-element abundance patterns in these two stars if absorption due to $\mathrm{Pb}$ II was of nearly equal strength in each one. There are many unidentified absorption features in the NUV spectra of these two stars, and we suspect that the residual absorption at $2203.53 \AA$ is probably not due to $\mathrm{Pb}$ II in HD 108317 or HD 128279. We derive an upper limit on the $\mathrm{Pb}$ abundance from this $\mathrm{Pb}$ II line assuming all of the residual absorption is due to $\mathrm{Pb}$ II.

\subsection{Bismuth}

We derive an upper limit on the $\mathrm{Bi}$ abundance from the Bi I resonance line at 2230.61 $\AA$ in HD 108317. As shown in Figure 7, this feature is weak, but Morton (2000) indicates that it should be among the strongest of all $\mathrm{Bi}$ I lines in late-type stars. Other species may also contribute to the absorption at this wavelength. To estimate the contribution from Bi I, we employ a technique we have used previously to estimate the absorption due to Au in HD 108317 (see Appendix A of Roederer et al. $2012 b$ ). Since the third $r$-process peak elements are not detected in HD 128279, we assume that all absorption at this wavelength 
Table 4

Mean Abundances of Species Examined in This Study

\begin{tabular}{|c|c|c|c|c|c|c|c|c|c|c|c|c|}
\hline \multirow[t]{2}{*}{ Species } & \multirow[t]{2}{*}{$Z$} & \multirow[t]{2}{*}{$\log \epsilon_{\odot}$} & \multicolumn{5}{|c|}{ HD 108317} & \multicolumn{5}{|c|}{ HD 128279} \\
\hline & & & $\log \epsilon$ & {$[\mathrm{X} / \mathrm{Fe}]$} & $\sigma_{\text {stat }}$ & $\sigma_{\text {total }}$ & $N$ & $\log \epsilon$ & {$[\mathrm{X} / \mathrm{Fe}]$} & $\sigma_{\text {stat }}$ & $\sigma_{\text {total }}$ & $N$ \\
\hline $\mathrm{Cu}$ II & 29 & 4.19 & +1.42 & -0.40 & 0.22 & 0.28 & 4 & +1.36 & -0.37 & 0.23 & 0.29 & 3 \\
\hline $\mathrm{Ge} \mathrm{I}_{\mathrm{I}}$ & 32 & 3.65 & +0.19 & -0.93 & 0.14 & 0.18 & $4^{\mathrm{a}}$ & -0.03 & -1.20 & 0.25 & 0.28 & $3^{a}$ \\
\hline As I & 33 & 2.30 & -0.30 & -0.07 & 0.56 & 0.57 & 1 & -0.49 & -0.31 & 0.49 & 0.50 & 1 \\
\hline Se I & 34 & 3.34 & +1.28 & +0.47 & 0.40 & 0.42 & 2 & +0.55 & -0.31 & 0.34 & 0.36 & 2 \\
\hline Mo II & 42 & 1.88 & -0.05 & +0.44 & 0.28 & 0.33 & 3 & -0.56 & +0.02 & 0.22 & 0.28 & 3 \\
\hline $\mathrm{Te}_{\mathrm{I}}$ & 52 & 2.18 & +0.06 & +0.41 & 0.28 & 0.30 & $2^{\mathrm{a}}$ & -0.12 & +0.18 & 0.27 & 0.30 & $2^{\mathrm{a}}$ \\
\hline $\mathrm{Yb}$ II & 70 & 0.92 & -1.13 & +0.32 & 0.14 & 0.22 & $1^{\mathrm{a}}$ & -1.69 & -0.15 & 0.13 & 0.21 & $1^{\mathrm{a}}$ \\
\hline $\mathrm{W}_{\text {II }}$ & 74 & 0.65 & $<-0.80$ & $<+0.92$ & $\ldots$ & $\ldots$ & 2 & $<-0.68$ & $<+1.13$ & $\ldots$ & $\ldots$ & 2 \\
\hline Os II & 76 & 1.40 & -0.68 & +0.29 & 0.32 & 0.36 & 2 & $<-1.28$ & $<-0.22$ & $\ldots$ & $\ldots$ & 2 \\
\hline Pt I & 78 & 1.62 & -0.40 & +0.51 & 0.15 & 0.19 & $5^{\mathrm{a}}$ & $<-0.78$ & $<+0.08$ & $\ldots$ & $\ldots$ & $5^{\mathrm{a}}$ \\
\hline $\mathrm{Hg}$ II & 80 & 1.17 & $<+0.30$ & $<+1.50$ & $\ldots$ & $\ldots$ & 1 & $<+0.53$ & $<+1.82$ & $\ldots$ & $\ldots$ & 1 \\
\hline
\end{tabular}

Notes. The $[\mathrm{X} / \mathrm{Fe}]$ ratios, where $\mathrm{X}$ represents one of the heavy elements of interest, are calculated by comparing neutrals with neutrals and ions with ions.

${ }^{a}$ The mean abundance or upper limit includes one or more lines from Roederer et al. (2012b).

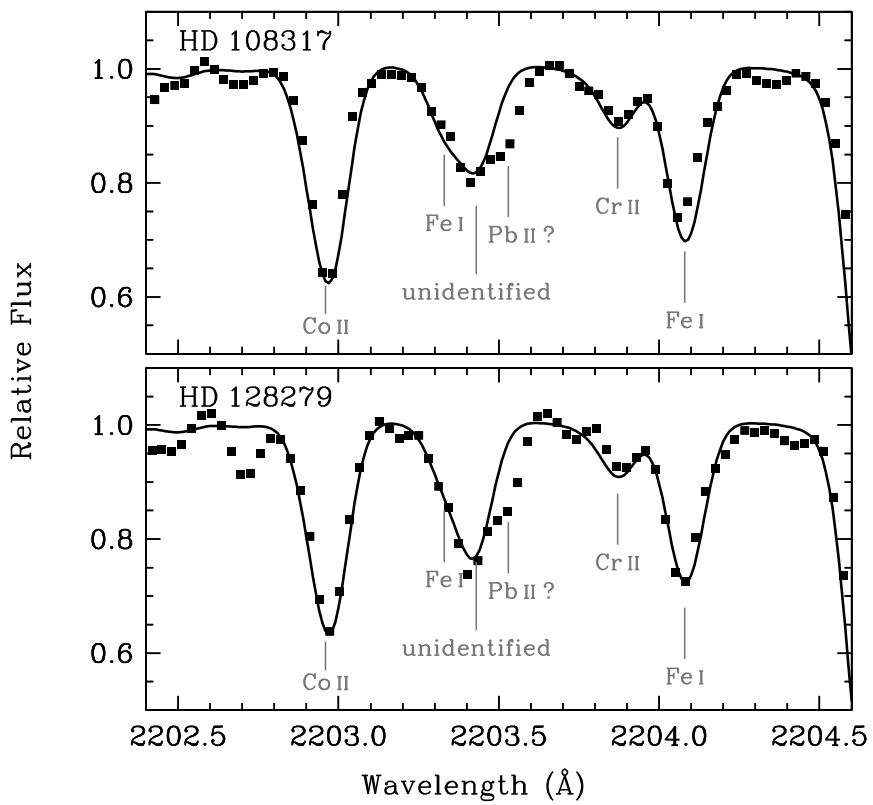

Figure 6. Comparison of observed and synthetic spectra around the $\mathrm{Pb}$ II line in HD 108317 and HD 128279. The filled squares mark the observed spectra, and the curves indicate a synthesis with no Pb II absorption. To facilitate comparisons, the label locations on the plots are identical in the top and bottom panels.

in $\mathrm{HD} 128279$ is not due to $\mathrm{Bi}$, and we adjust our line list to fit the observed line profile in HD 128279. We then use this line list to fit the absorption features in HD 108317. The fit is not satisfactory, so we cannot state with confidence that the $\mathrm{Bi}$ I line is detected. Figure 7 illustrates our derived upper limit.

\section{RESULTS}

Table 3 lists the abundances or upper limits derived from each line. Table 4 lists the mean abundances or upper limits for each species. The means include lines from other NUV transitions at longer wavelengths, when available. Values in Table 4 supersede those published previously by Roederer et al. (2012a, 2012b).

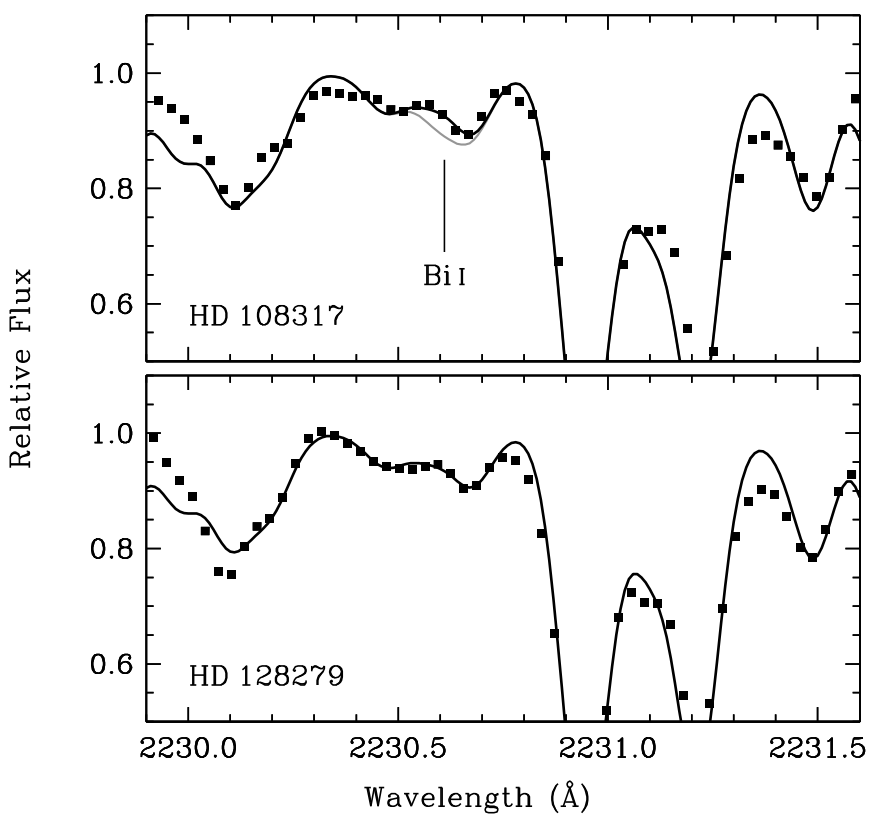

Figure 7. Comparison of observed and synthetic spectra around the Bi I line in HD 108317 and HD 128279. The filled squares mark the observed spectra. The black lines indicate a synthesis with no Bi I absorption, and the gray line in the top panel indicates our upper limit on the Bi abundance.

\subsection{Summary of Statistical Uncertainties}

The means in Table 4 are weighted by the statistical uncertainty obtained from the quadrature sum of the fitting uncertainty (Table 3), uncertainty in the $\log g f$ values (Table 3), and the uncertainty introduced by the wavelength-dependent corrections (Table 2). The uncertainties in Table 3 reflect fitting uncertainties estimated during the spectrum-synthesis matching. The main sources of error are continuum placement and blending features whose strength cannot be empirically set by fitting the observed line profile. These uncertainties are typically larger than those estimated for optical data. 
Table 5

Comparison of $[\mathrm{X} / \mathrm{Fe}]$ Ratios of Heavy Elements Derived from Neutral and Ionized Species

\begin{tabular}{|c|c|c|}
\hline Species & HD 108317 & HD 128279 \\
\hline $\mathrm{Cu} \mathrm{I}$ & $-0.96 \pm 0.16$ & $-0.92 \pm 0.16$ \\
\hline $\mathrm{Cu} \mathrm{II}$ & $-0.40 \pm 0.28$ & $-0.37 \pm 0.29$ \\
\hline $\mathrm{Zn}$ I & $+0.23 \pm 0.15$ & $+0.10 \pm 0.15$ \\
\hline $\mathrm{Zn} \mathrm{II}$ & $+0.31 \pm 0.52$ & $+0.28 \pm 0.44$ \\
\hline Mo I & $+0.42 \pm 0.19$ & $-0.07 \pm 0.18$ \\
\hline Mo II & $+0.44 \pm 0.33$ & $+0.02 \pm 0.28$ \\
\hline $\mathrm{Cd}_{\mathrm{I}}$ & $-0.39 \pm 0.46$ & $-0.57 \pm 0.36$ \\
\hline Cd II & $-0.42 \pm 0.52$ & $-0.55 \pm 0.43$ \\
\hline Os I & $+0.63 \pm 0.23$ & $<+0.69$ \\
\hline Os II & $+0.29 \pm 0.36$ & $<-0.22$ \\
\hline $\mathrm{Pb}$ & $<+0.23$ & $<+0.35$ \\
\hline $\mathrm{Pb}$ II & $<+0.42$ & $<+0.54$ \\
\hline
\end{tabular}

\subsection{Systematic Uncertainties}

Systematic uncertainties resulting from errors in the model atmosphere parameters have been discussed previously (Cowan et al. 2005), and we adopt their assessment of the uncertainties. This uncertainty amounts to 0.12 dex for lines of neutral species and 0.17 dex for lines of ionized species. These uncertainties are added in quadrature with the statistical errors listed in Table 4 to form the total errors listed there.

We detect multiple transitions of many of the species examined in our study. For Cu II, Ge I, Se I, Mo II, Te I, and Pt I, the abundances derived from multiple transitions are in good agreement. These values are listed in Table 3 in the present study and Table 3 of Roederer et al. (2012b).

We detect neutral and singly ionized states of $\mathrm{Cu}, \mathrm{Zn}$, Mo, $\mathrm{Cd}$, and Os. The neutral species of $\mathrm{Cu}, \mathrm{Zn}$, and Mo were detected from optical transitions by Roederer et al. (2012b). These abundances are listed in Table 5. The $[\mathrm{Zn} / \mathrm{Fe}],[\mathrm{Mo} / \mathrm{Fe}]$, $[\mathrm{Cd} / \mathrm{Fe}]$, and $[\mathrm{Os} / \mathrm{Fe}]$ ratios derived from neutral and ionized atoms agree, although the uncertainties are substantial in some cases. Nevertheless, this places limits on the magnitude of overionization or other non-LTE effects that could occur for the transitions studied in these two stars.

In contrast, the $[\mathrm{Cu} / \mathrm{Fe}]$ ratio derived from ions is higher than that derived from the neutral species in HD 108317 and HD 128279: $\langle[\mathrm{Cu}$ II $/ \mathrm{Cu}$ I $]\rangle=+0.56 \pm 0.23$. For completeness, we note that Roederer \& Lawler (2012) found a difference of $+0.29 \pm 0.35 \mathrm{dex}$ in the $[\mathrm{Cu} / \mathrm{Fe}]$ ratios derived from $\mathrm{Cu}$ II and $\mathrm{I}$ lines in the warm $(5950 \mathrm{~K})$ metal-poor $([\mathrm{Fe} / \mathrm{H}]=-1.8)$ subgiant HD 160617. Most $\mathrm{Cu}$ atoms are ionized in these stellar atmospheres, so overionization of $\mathrm{Cu}$ I relative to the LTE populations could occur. The $\mathrm{Cu}$ I abundances in these three stars are derived from the 3247 and $3273 \AA$ resonance lines. Bonifacio et al. (2010) found that these lines did not give consistent results with the Cu I 5105 and $5782 \AA$ lines in dwarfs and giants in two metal-poor globular clusters. Their 3D LTE modeling of the line formation could not fully reconcile the abundances, and it was not apparent which set of lines (if either) could be reliable $\mathrm{Cu}$ abundance indicators given the current state of modeling tools. Our results further suggest that $\mathrm{Cu}$ I lines may not be formed in LTE, but clearly more work is needed to better understand the formation of $\mathrm{Cu}$ lines in cool stars.

Roederer et al. (2012b) discussed the possible discrepancy between the $[\mathrm{Os} / \mathrm{Fe}]$ ratios derived from Os I and II lines in HD 108317: the Os I $3058 \AA$ line yields an abundance ( $[\mathrm{Os} / \mathrm{Fe}]=+0.63 \pm 0.23$ ) higher than that given by the Os II $2282 \AA$ line $([\mathrm{Os} / \mathrm{Fe}]=+0.14 \pm 0.42)$. Roederer et al. (2010b) reported an abundance difference of $+0.34 \pm 0.30$ dex between the Os I $3058 \AA$ line and the Os II $2282 \AA$ line in the metalpoor giant $\mathrm{BD}+173248$. Each difference is not significant given the uncertainties, and other results suggest these differences are not symptoms of systematic uncertainties. Cowan et al. (2005) found that the Os I $3058 \AA$ line gave concordant results with the Os I 2838 and $3301 \AA$ lines $(\sigma=0.08$ dex $)$ in $\mathrm{BD}+17$ 3248. Ivarsson et al. (2004) found that the Os II $2282 \AA$ line also gave concordant results with the Os II 2067 and $2070 \AA$ lines $(\sigma=0.1 \mathrm{dex})$ in the chemically peculiar star $\chi$ Lupi. The extant data do not indicate systematic differences between the Os abundance indicators, but reducing the statistical uncertainties and examining abundances derived from these lines in additional stars would provide worthwhile checks.

As shown in Table 5, the upper limits on $[\mathrm{Pb} / \mathrm{Fe}]$ derived from the $\mathrm{Pb}$ II $2203 \AA$ line are slightly higher than the strongest upper limits on $[\mathrm{Pb} / \mathrm{Fe}]$ derived from the $\mathrm{Pb}$ I $2833 \AA$ line (Roederer et al. 2012b). Roederer et al. (2010a) reported a detection of the $\mathrm{Pb}$ I $4057 \AA$ line in HD 108317 based on a higher-quality spectrum taken with the Tull Spectrograph (Tull et al. 1995) on the Smith Telescope at McDonald Observatory. The line is weak, and that detection is tenuous. If it is indeed a detection, however, we would derive $[\mathrm{Pb} / \mathrm{Fe}] \approx+0.1 \pm 0.3$ using our model atmosphere for HD 108317. The Mashonkina et al. (2012) non-LTE correction to the $\mathrm{Pb}$ abundance derived from the $\mathrm{Pb}$ I $4057 \AA$ line implies $[\mathrm{Pb} / \mathrm{Fe}] \approx+0.62 \pm 0.3$. If the $[\mathrm{Pb} / \mathrm{Fe}]$ derived in LTE from $\mathrm{Pb}$ II lines $(<+0.42)$ accurately reflects the $\mathrm{Pb}$ abundance, then our upper limit is $\approx 0.2$ dex lower than the value predicted by the non-LTE corrections. This difference is within the uncertainties, so it does not justify revision of the non-LTE corrections or rejection of the detection.

\section{DISCUSSION}

\subsection{The $[\mathrm{As} / \mathrm{Fe}]$ and $[\mathrm{Se} / \mathrm{Fe}]$ Ratios}

Figure 8 illustrates the $[\mathrm{As} / \mathrm{Fe}]$ and $[\mathrm{Se} / \mathrm{Fe}]$ ratios in $\mathrm{HD}$ 108317, HD 128279, and seven other stars where As and Se have been studied. The data show no compelling evidence for a cosmic dispersion in either $[\mathrm{As} / \mathrm{Fe}]$ or $[\mathrm{Se} / \mathrm{Fe}]$. On average, both ratios are slightly super-solar over the entire metallicity range considered. The mean $[\mathrm{As} / \mathrm{Fe}]$ and $[\mathrm{Se} / \mathrm{Fe}]$ ratios are $+0.28 \pm 0.14(\sigma=0.36 \mathrm{dex})$ and $+0.16 \pm 0.09(\sigma=0.26 \mathrm{dex})$, respectively. In these expressions of uncertainties, the former value (e.g., $0.14 \mathrm{dex}$ ) is the standard error of the mean, and the latter value (e.g., $0.36 \mathrm{dex}$ ) is the sample standard deviation.

Figure 8 also illustrates the [Ge/Fe] ratios in HD 108317 , HD 128279, and 17 other stars where Ge has been detected. There is relatively little change in the $[\mathrm{Ge} / \mathrm{Fe}]$ ratio for stars with metallicities in the range $-3.1<[\mathrm{Fe} / \mathrm{H}]<-1.4$, a fact first noticed by Cowan et al. (2005). That study also noticed that $[\mathrm{Ge} / \mathrm{Fe}]$ showed no correlation with $[\mathrm{Eu} / \mathrm{Fe}]$ for the 10 stars in their sample. Cowan et al. concluded that Ge production in the early Galaxy was decoupled from the $r$-process nucleosynthesis responsible for heavy elements, such as europium (Eu, $Z=63$ ).

In contrast with the $[\mathrm{As} / \mathrm{Fe}]$ and $[\mathrm{Se} / \mathrm{Fe}]$ ratios, the mean $[\mathrm{Ge} / \mathrm{Fe}]$ ratio in stars with $[\mathrm{Fe} / \mathrm{H}]<-1.4$ is $-0.91 \pm 0.07(\sigma=$ $0.26 \mathrm{dex})$. At higher metallicities, the $[\mathrm{Ge} / \mathrm{Fe}]$ ratio increases toward the solar ratio, whereas $[\mathrm{As} / \mathrm{Fe}]$ and $[\mathrm{Se} / \mathrm{Fe}]$ do not show a similar increase. Roederer (2012) tentatively attributed the increase in $[\mathrm{Ge} / \mathrm{Fe}]$ to the weak component of the $s$-process 


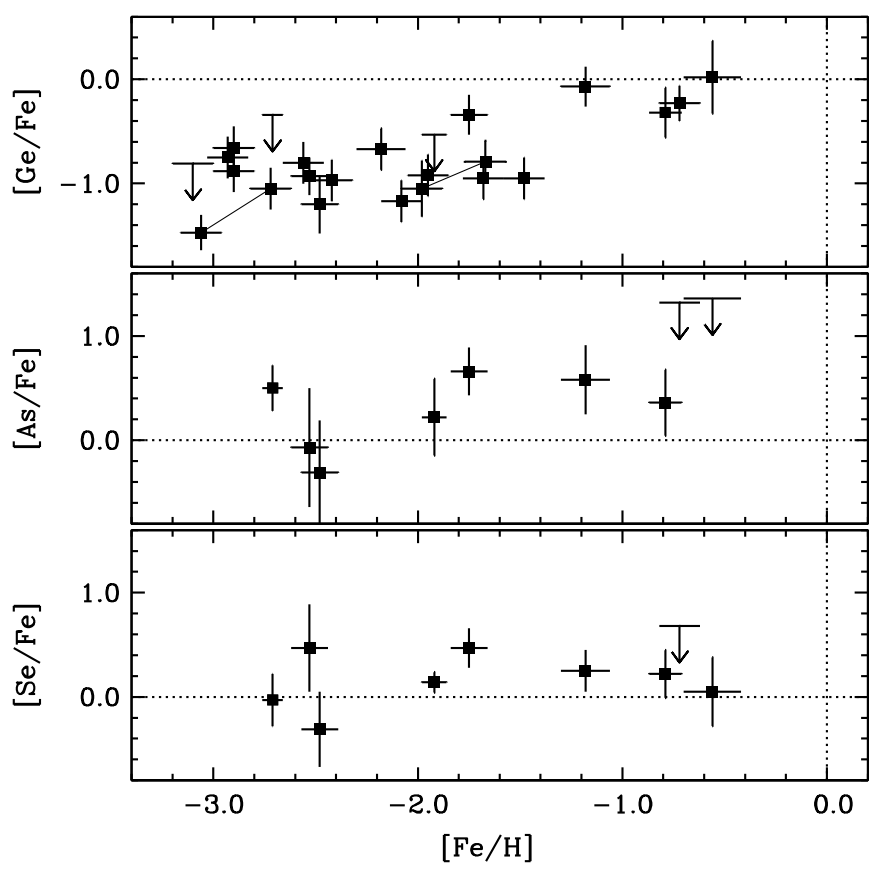

Figure 8. $[\mathrm{Ge} / \mathrm{Fe}],[\mathrm{As} / \mathrm{Fe}]$, and $[\mathrm{Se} / \mathrm{Fe}]$ ratios for all late-type stars with reported $\mathrm{Ge}, \mathrm{As}$, or Se abundances. The dotted lines represent the solar ratios. The $[\mathrm{Fe} / \mathrm{H}]$ values shown are derived from optical $\mathrm{Fe}$ I lines. This figure includes abundances from Sneden et al. (1998), Cowan et al. (2002, 2005), Hill et al. (2002), Ivans et al. (2006), Roederer (2012), Roederer \& Lawler (2012), Roederer et al. (2012b), Siqueira Mello et al. (2013), and the present study. The thin lines in the $[\mathrm{Ge} / \mathrm{Fe}]$ panel connect the abundances reported by Roederer et al. (2012b) to Cowan et al. (1996, 2005) or Sneden et al. (1998) for HD 122563 and HD 126238. All [Ge/Fe] ratios have been corrected to the NIST $\log g f$ abundance scale.

(see also Pignatari et al. 2010), which does not appear to have contributed noticeable amounts of As or Se to these stars.

\subsection{The Heavy Element Abundance Patterns}

Figure 9 combines our results with those of Roederer et al. (2012b) to illustrate the heavy-element abundance patterns of HD 108317 and HD 128279. The solar system $r$-process and $s$-process patterns (Sneden et al. 2008) are shown as fiducials for comparison. The bottom panels of Figure 9 illustrate the differences between the derived abundances in each star and the solar $r$-process residuals when normalized to Eu. A relatively smooth pattern emerges among the differences in HD 108317. The Ge, As, and Se $(32 \leqslant Z \leqslant 34)$ abundances in HD 108317 exhibit an upward trend relative to the scaled solar system $r$-process component. This trend flattens for $\mathrm{Sr}$ through $\mathrm{Mo}$ $(38 \leqslant Z \leqslant 42)$, and the differences then smoothly decrease for $\mathrm{Ru}$ through $\mathrm{Cd}(44 \leqslant Z \leqslant 48)$. There may be a gentle increase from $\mathrm{Cd}$ through $\mathrm{Ba}(48 \leqslant Z \leqslant 56)$, indicated by Te, and possibly extending through $\operatorname{Pr}(Z=59)$. Otherwise, the abundances of $\mathrm{Ba}$ through $\mathrm{Lu}(56 \leqslant Z \leqslant 71)$ closely follow the scaled solar system $r$-process pattern. This close correspondence may extend to Os and $\operatorname{Pt}(Z=76$ and 78$)$ at the $3 \mathrm{rd} r$-process peak. There are several anomalous elements that do not follow this general pattern (Y, $Z=39$; Hf, $Z=72$; Au, $Z=79$ ), but overall there is a relatively smooth pattern among the differences.

Similar trends are observed in HD 128279, as shown in Figure 9, but there is more element-to-element scatter obscuring them. The upper limits on Os and Pt at the 3rd $r$-process peak indicate a downturn in the abundances in HD 128279 relative to the scaled solar system $r$-process pattern.

\subsection{The Second r-process Peak}

There is ample observational evidence for the existence of at least two components of $r$-process nucleosynthesis (the so-called main and weak components) operating in the early Galaxy (e.g., McWilliam 1998; Johnson \& Bolte 2002; Aoki et al. 2005; Qian \& Wasserburg 2008; Hansen et al. 2012). Elements through $\mathrm{Cd}(A \leqslant 116)$ are commonly associated with the weak component, and the consistent $r$-process abundance pattern found for $\mathrm{Ba}$ and the rare-earth elements $(A \geqslant 135)$ is a defining characteristic of the main component.

Te $(125 \leqslant A \leqslant 130)$, an element at the second $r$-process peak, has been detected in four stars: BD+17 3248, HD 108317, HD 128279, and HD 160617 (Roederer \& Lawler 2012; Roederer et al. 2012a, 2012b; this study). The dispersions
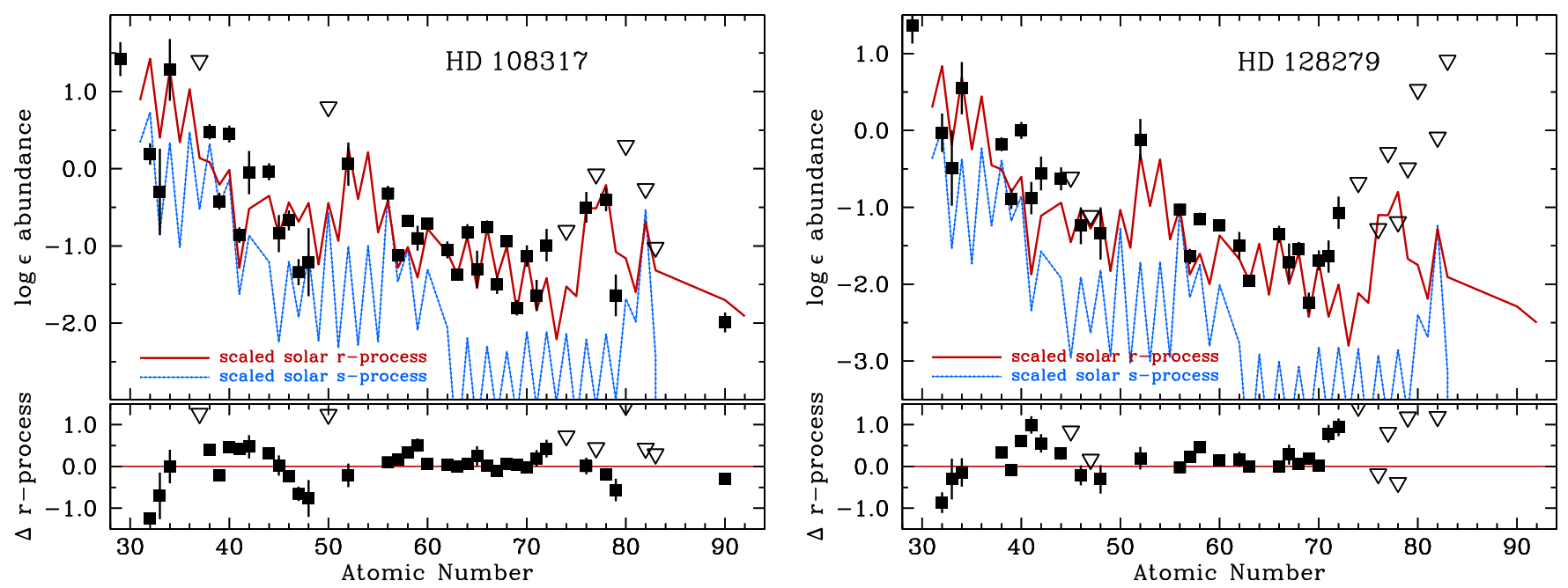

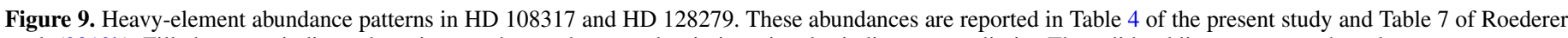

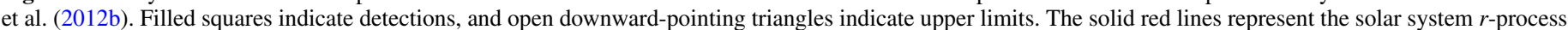

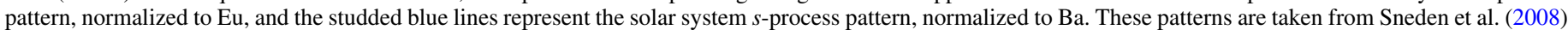

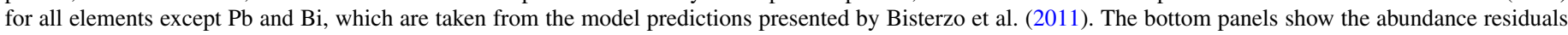
with respect to the $r$-process pattern.

(A color version of this figure is available in the online journal.) 
among the ratios of $\mathrm{Te}$ and lighter elements ([Te/ $\mathrm{Ru}]$, $[\mathrm{Te} / \mathrm{Pd}],[\mathrm{Te} / \mathrm{Ag}]$, and $[\mathrm{Te} / \mathrm{Cd}])$ are $0.14,0.19,0.08$, and 0.28 dex, respectively. The dispersions among the ratios of $\mathrm{Te}$ and heavier elements ([Te/Ba], $[\mathrm{Te} / \mathrm{La}],[\mathrm{Te} / \mathrm{Ce}]$, and $[\mathrm{Te} / \mathrm{Nd}])$ are $0.31,0.16,0.12$, and 0.13 dex, respectively. There is no obvious preference for smaller dispersions among Te and lighter or heavier element groups, which might be expected if Te was predominantly produced in one of the weak or main components. We interpret these data to indicate that Te owes its nucleosynthesis to conditions intermediate between the main and weak components.

\subsection{The First r-process Peak}

Figure 9 indicates that the Se abundances in HD 108317 and HD 128279 are a good match to the solar system $r$-process residuals when normalized to Eu. We can estimate the percentage of Se that originated in the main and weak components of the $r$-process. We need to consider only the differential abundance ratios in HD 108317 and HD 128279 because the stellar parameters of these two stars are so similar.

We subtract the main component of the $r$-process pattern (given by the star CS 22892-052; Sneden et al. 2003, 2009), normalized to Eu, from both HD 108317 and HD 128279. The residual abundance pattern is attributed to the weak component of the $r$-process (see Montes et al. 2007). The weak-to-main ratio in HD 108317 is $3.7 \pm 0.5$ times greater than in HD 128279 for elements between the first and second $r$-process peaks. The ratio of the $\mathrm{Se} / \mathrm{Eu}$ abundance ratios in HD 108317 and HD 128279 would be low (1.0) if the Se originated only in the main component of $r$-process, and it would be high (3.7) if the Se originated only in the weak component. The observed ratio of the $\mathrm{Se} / \mathrm{Eu}$ ratios is $\approx 1.4_{-0.7}^{+1.4}$. This is compatible with $1.0 \mathrm{but}$ is mildly incompatible with $3.7 \pm 0.5$, hinting that a portion of the Se could have originated in the main $r$-process. The $1 \sigma$ upper limit of 2.8 on the ratio of $\mathrm{Se} / \mathrm{Eu}$ ratios translates into a lower limit of the contribution from the main $r$-process to Se of about $12 \% \pm 4 \%$.

Tighter observational uncertainties are the key to constraining this value better and disentangling the weak and main $r$-process contributions to the elements in the $A \approx 80$ mass region. Great effort by experimentalists has provided much of the necessary nuclear data (masses, decay half-lives, and $\beta$-delayed neutron emission) for each of the isotopes in this mass region relevant for $r$-process nucleosynthesis (e.g., Baruah et al. 2008; Hosmer et al. 2010). Observers must provide a suitable set of observational constraints for $r$-process models that make use of these data. The solar system $r$-process residuals implicitly include contributions from all processes except for the $s$-process, yet Farouqi et al. (2010) and others have pointed out that the $r$-process residuals in the $A \lesssim 90$ mass regime are challenging to interpret because several nucleosynthetic processes contribute to them. Alternate template $r$-process patterns for this mass regime are desirable. The abundance pattern for elements with $32 \leqslant Z \leqslant 48$ in HD 108317 may present an alternative. The low metallicity of this star indicates that its metals derive from relatively few enrichment events. This star has received no or minimal $s$-process contributions. The detection of Se, an element at the first $r$-process peak, in this star is an essential step in the right direction.

\subsection{The Beginning of Nucleosynthesis beyond the Iron Group}

The Ge-As-Se trio of elements has been studied in three metal-poor stars: HD 108317, HD 128279, and HD 160617.

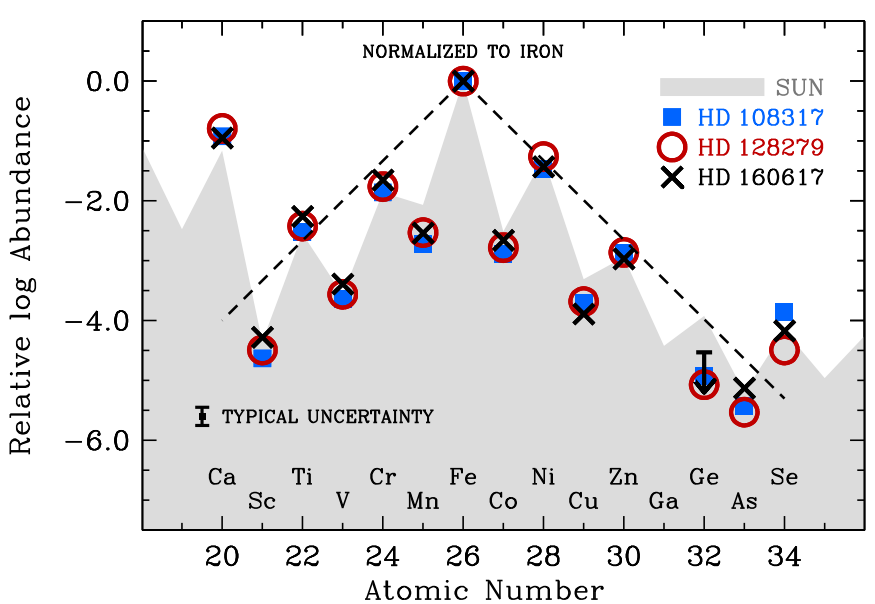

Figure 10. Abundances at the iron peak in the Sun and three metal-poor stars. All abundance patterns are normalized to $\log (\mathrm{Fe})=0$. The dashed lines are intended to guide the eye to approximately connect the abundances of even- $Z$ elements. Note that the Se abundances in these stars are enhanced relative to a simple extrapolation of the even- $Z$ iron-peak abundances.

(A color version of this figure is available in the online journal.)

Abundances of elements in the iron group have also been reported for these three stars by Roederer \& Lawler (2012) and Roederer et al. (2014). These abundances are illustrated in Figure 10. The solar abundances are shown for comparison. Each pattern is normalized to Fe. The dashed lines in Figure 10 trace the abundances of the even-numbered elements in the iron group.

In massive stars, the iron-group elements are formed during explosive ${ }^{28} \mathrm{Si}$ burning, and this produces the familiar shape of the iron peak. At the light end of the iron peak, $\mathrm{Ca}$ in the Sun and these three stars is $>3$ dex more abundant than the simple extrapolation of the even- $Z$ iron peak abundances would predict for $Z=20$. This results from a different, more efficient mechanism of producing ${ }^{40} \mathrm{Ca}, \alpha$-capture on the products of ${ }^{16} \mathrm{O}$ burning. At the heavy end of the iron peak, an extrapolation of the dashed line to elements heavier than $\mathrm{Zn}$ reveals that the stellar Ge abundances fall $\approx 1$ dex lower than the extrapolation, and the stellar Se abundances fall $\approx 1$ dex higher than the extrapolation These values disagree with the extrapolation by $\approx 2.5-3.5$ times the abundance uncertainties. Using the $\mathrm{Ca}$ overabundance to guide our interpretation of $\mathrm{Ge}$ and $\mathrm{Se}$, we speculate that the stellar deficiency of $\mathrm{Ge}$ and excess of Se indicate the mass regime $(75<A \leqslant 82)$ where a different nucleosynthetic mechanism dominates the quasi-equilibrium $\alpha$-rich freezeout of the iron peak.

In general, $r$-process models predict that these elements are produced by charged-particle reactions, neutron-capture reactions, or both. Both $v p$ reactions and $\alpha$-rich freezeout produce low- $A$, neutron-deficient isotopes (Fröhlich et al. 2006; Pruet et al. 2006), while neutron-capture reactions produce high- $A$, neutron-rich isotopes (Woosley \& Hoffman 1992; Woosley et al. 1994; Farouqi et al. 2010). The fact that the excess occurs at $\mathrm{Se}(A \approx 80)$ may provide a clue to the dominant nucleosynthesis mechanism. Nuclei in this mass region could be the stable descendants of unstable nuclei at the neutron-magic $N=$ 50 closed shell along the $r$-process path, hinting at an $r$-process origin. Isotopic abundances of $\mathrm{Ge}$ and Se would resolve the issue definitively, but measurements of isotopic ratios are not likely to be available in the foreseeable future. Ge and Se have small hfs and IS, making such measurements difficult if not impossible, and in any case the NUV spectroscopic observations that would 
be required are prohibitively long. Two practical approaches to better understand the astrophysical origins of these nuclei may be to derive elemental abundances from larger samples of stars with diverse heavy-element abundance patterns and to reduce the statistical uncertainties by acquiring higher-quality spectra when possible.

\section{SUMMARY}

We have obtained new high-resolution, high-S/N NUV spectroscopic observations of the metal-poor giants HD 108317 and HD 128279. These observations extend the high-quality spectral coverage of HD 108317 and HD 128279 from $\lambda<2000 \AA$ to nearly $1 \mu \mathrm{m}$. We have derived new abundances or upper limits from 27 lines of elements with $Z \geqslant 29$ in these stars.

We have derived $[\mathrm{Cu} / \mathrm{Fe}]$ from both neutral and ionized species, and $\langle[\mathrm{Cu}$ II $/ \mathrm{Cu} \mathrm{I}]\rangle=+0.56 \pm 0.23$ in $\mathrm{HD} 108317$ and HD 128279. These data hint that $\mathrm{Cu}$ I may not be formed in LTE in these stars. We are not aware of any non-LTE calculations for $\mathrm{Cu}$ in late-type stars. We echo the caution issued by Bonifacio et al. (2010) that $\mathrm{Cu}$ abundances derived from $\mathrm{Cu}$ I lines in metalpoor stars should be treated with caution until such calculations become available.

The $[\mathrm{Zn} / \mathrm{Fe}],[\mathrm{Mo} / \mathrm{Fe}],[\mathrm{Cd} / \mathrm{Fe}]$, and $[\mathrm{Os} / \mathrm{Fe}]$ ratios have been derived from both neutral and ionized species. In these cases the ratios from neutrals and ions agree within the uncertainties, which span 0.15-0.52 dex (see Table 5). These data also corroborate the $\mathrm{Ge}, \mathrm{Cd}, \mathrm{Te}, \mathrm{Os}$, and $\mathrm{Pt}$ abundances derived from other lines in HD 108317 and HD 128279 (Roederer et al. 2012a, 2012b).

Comparison of the As and Se abundances with those of iron-group elements indicates that the mass region between Ge and Se $(75 \leqslant A \leqslant 82)$ may identify the mass at which a different nucleosynthetic mechanism begins to dominate the quasi-equilibrium $\alpha$-rich freezeout of the iron peak.

The data hint that a portion $(\gtrsim 10 \%)$ of the Se could have originated in the main component of the $r$-process. Reducing both the statistical and systematic uncertainties on Se and increasing the sample of stars with precise As and Se measurements should remain high priorities while $H S T$ is operational.

We thank the referee for providing helpful comments on this manuscript. This research has made use of NASA's Astrophysics Data System Bibliographic Services, the arXiv pre-print server operated by Cornell University, the SIMBAD and VizieR databases hosted by the Strasbourg Astronomical Data Center, the ASD hosted by NIST, and the Mikulski Archive at the Space Telescope Science Institute. IRAF is distributed by the National Optical Astronomy Observatories, which are operated by the Association of Universities for Research in Astronomy, Inc., under cooperative agreement with the National Science Foundation. Generous support for Programs GO-12268 and GO-12976 was provided by NASA through grants from the Space Telescope Science Institute, which is operated by the Association of Universities for Research in Astronomy, Incorporated, under NASA contract NAS 5-26555. H.S. acknowledges support from grant PHY 11-0251 from the US National Science Foundation. H.S. and T.C.B. acknowledge partial support for this work from grant PHY 08-22648; Physics Frontier Center/Joint Institute for Nuclear Astrophysics (JINA), awarded by the US National Science Foundation. A.F. acknowledges support from grant AST 12-55160 from the US National Science Foundation.

Facility: HST (STIS)

\section{REFERENCES}

Alonso, A., Arribas, S., \& Martínez-Roger, C. 1999, A\&AS, 140, 261

Aoki, W., Honda, S., Beers, T. C., et al. 2005, ApJ, 632, 611

Barbuy, B., Spite, M., Hill, V., et al. 2011, A\&A, 534, A60

Baruah, S., Audi, G., Blaum, K., et al. 2008, PhRvL, 101, 262501

Bergeson, S. D., \& Lawler, J. E. 1993, ApJ, 408, 382

Bisterzo, S., Gallino, R., Straniero, O., Cristallo, S., \& Käppeler, F. 2011, MNRAS, 418, 284

Bonifacio, P., Caffau, E., \& Ludwig, H.-G. 2010, A\&A, 524, A96

Castelli, F., \& Kurucz, R. L. 2004, in IAU Symp. 210, Modelling of Stellar Atmospheres, ed. N. Piskunov et al. (Cambridge: Cambridge Univ. Press), A20

Cowan, J. J., Sneden, C., Beers, T. C., et al. 2005, ApJ, 627, 238

Cowan, J. J., Sneden, C., Burles, S., et al. 2002, ApJ, 572, 861

Cowan, J. J., Sneden, C., Truran, J. W., \& Burris, D. L. 1996, ApJL, 460, L115

Den Hartog, E. A., Herd, M. T., Lawler, J. E., et al. 2005, ApJ, 619, 639

Farouqi, K., Kratz, K.-L., Pfeiffer, B., et al. 2010, ApJ, 712, 1359

Fröhlich, C., Martínez-Pinedo, G., Liebendörfer, M., et al. 2006, PhRvL, 96, 142502

Hansen, C. J., Primas, F., Hartman, H., et al. 2012, A\&A, 545, A31

Hill, V., Plez, B., Cayrel, R., et al. 2002, A\&A, 387, 560

Hosmer, P., Schatz, H., Aprahamian, A., et al. 2010, PhRvC, 82, 025806

Ito, H., Aoki, W., Beers, T. C., et al. 2013, ApJ, 773, 33

Ivans, I. I., Simmerer, J., Sneden, C., et al. 2006, ApJ, 645, 613

Ivarsson, S., Wahlgren, G. M., Dai, Z., Lundberg, H., \& Leckrone, D. S. 2004, A\&A, 425, 353

Johnson, J. A., \& Bolte, M. 2002, ApJ, 579, 616

Kimble, R. A., Woodgate, B. E., Bowers, C. W., et al. 1998, ApJL, 492, L83

Kramida, A., Ralchenko, Yu., Reader, J., et al. 2013, NIST Atomic Spectral Database (ver. 4.0-5.1) (Gaithersburg, MD: National Institute of Standards and Technology), available at http://physics.nist.gov/asd

Lawler, J. E., Guzman, A., Wood, M. P., Sneden, C., \& Cowan, J. J. 2013, ApJS, 205,11

Luck, R. E., \& Bond, H. E. 1985, ApJ, 292, 559

Mashonkina, L., Ryabtsev, A., \& Frebel, A. 2012, A\&A, 540, A98

McWilliam, A. 1998, AJ, 115, 1640

Migdałek, J. 1983, JQSRT, 30, 169

Montes, F., Beers, T. C., Cowan, J., et al. 2007, ApJ, 671, 1685

Morton, D. C. 2000, ApJS, 130, 403

Nilsson, H., Engström, L., Lundberg, H., et al. 2008, EPJD, 49, 13

Peterson, R. C. 2011, ApJ, 742, 21

Pignatari, M., Gallino, R., Heil, M., et al. 2010, ApJ, 710, 1557

Placco, V. M., Beers, T. C., Roederer, I. U., et al. 2014, ApJ, 790, 34

Pruet, J., Hoffman, R. D., Woosley, S. E., Janka, H.-T., \& Buras, R. 2006, ApJ, 644, 1028

Qian, Y.-Z., \& Wasserburg, G. J. 2008, ApJ, 687, 272

Quinet, P., Palmeri, P., Biémont, É., et al. 2006, A\&A, 448, 1207

Roederer, I. U. 2012, ApJ, 756, 36

Roederer, I. U., \& Lawler, J. E. 2012, ApJ, 750, 76

Roederer, I. U., Cowan, J. J., Karakas, A. I., et al. 2010a, ApJ, 724, 975

Roederer, I. U., Kratz, K.-L., Frebel, A., et al. 2009, ApJ, 698, 1963

Roederer, I. U., Lawler, J. E., Cowan, J. J., et al. 2012a, ApJL, 747, L8

Roederer, I. U., Lawler, J. E., Sobeck, J. S., et al. 2012b, ApJS, 203, 27

Roederer, I. U., Preston, G. W., Thompson, I. B., et al. 2014, AJ, 147, 136

Roederer, I. U., Sneden, C., Lawler, J. E., \& Cowan, J. J. 2010b, ApJL, 714, L123

Sikström, C. M., Pihlemark, H., Nilsson, H., et al. 2001, JPhB, 34, 477

Siqueira Mello, C., Spite, M., Barbuy, B., et al. 2013, A\&A, 550, A122

Sneden, C. A. 1973, PhD thesis, Univ. Texas at Austin

Sneden, C., Cowan, J. J., Burris, D. L., \& Truran, J. W. 1998, ApJ, 496, 235

Sneden, C., Cowan, J. J., \& Gallino, R. 2008, ARA\&A, 46, 241

Sneden, C., Cowan, J. J., Lawler, J. E., et al. 2003, ApJ, 591, 936

Sneden, C., Lawler, J. E., Cowan, J. J., Ivans, I. I., \& Den Hartog, E. A. 2009, ApJS, 182, 80

Sobeck, J. S., Kraft, R. P., Sneden, C., et al. 2011, AJ, 141, 175

Travaglio, C., Gallino, R., Arnone, E., et al. 2004, ApJ, 601, 864

Tull, R. G., MacQueen, P. J., Sneden, C., \& Lambert, D. L. 1995, PASP, 107,251

Wood, M. P., Lawler, J. E., Sneden, C., \& Cowan, J. J. 2013, ApJS, 208, 27

Wood, M. P., Lawler, J. E., Sneden, C., \& Cowan, J. J. 2014, ApJS, 211, 20

Woodgate, B. E., Kimble, R. A., Bowers, C. W., et al. 1998, PASP, 110, 1183

Woosley, S. E., \& Hoffman, R. D. 1992, ApJ, 395, 202

Woosley, S. E., Wilson, J. R., Mathews, G. J., Hoffman, R. D., \& Meyer, B. S. 1994, ApJ, 433, 229 